\title{
World Workshop on Oral Medicine VII: Targeting the oral microbiome Part 2: Current knowledge on malignant and potentially malignant oral disorders
}

\author{
Jairo Robledo-Sierra ${ }^{1}$ | Dalit Porat Ben-Amy ${ }^{2}$ | Elena Varoni ${ }^{3}$ | Roxanne Bavarian ${ }^{4,5}$ | \\ Janne L. Simonsen ${ }^{6}$ | Bruce J. Paster ${ }^{7}$ | William G. Wade ${ }^{8}$ | Alexander R. Kerr ${ }^{9}$ (D) | \\ Douglas E. Peterson ${ }^{10}$ | Ellen Frandsen Lau ${ }^{11}$ id \\ ${ }^{1}$ Faculty of Dentistry, CES University, Medellin, Colombia \\ ${ }^{2}$ Oral Medicine Unit, Department of Oral \& Maxillofacial Surgery, The Baruch Padeh Medical Center, Poriya, Israel \\ ${ }^{3}$ Department of Biomedical, Surgical and Dental Sciences, University of Milan, Milan, Italy \\ ${ }^{4}$ Division of Oral Medicine and Dentistry, Brigham and Women's Hospital, Boston, Massachusetts \\ ${ }^{5}$ Department of Oral Medicine, Infection, and Immunity, Harvard School of Dental Medicine, Harvard University, Cambridge, Massachusetts \\ ${ }^{6}$ Aarhus University Library - Health Sciences, Aarhus University, Aarhus, Denmark \\ ${ }^{7}$ The Forsyth Institute, Cambridge, Massachusetts \\ ${ }^{8}$ Centre for Host-Microbiome Interactions, Faculty of Dentistry, Oral \& Craniofacial Sciences, King's College London, London, UK \\ ${ }^{9}$ Department of Oral and Maxillofacial Pathology, Radiology and Medicine, New York University College of Medicine, New York City, New York \\ ${ }^{10}$ Oral Medicine Section, School of Dental Medicine, UConn Health, University of Connecticut, Mansfield, Connecticut \\ ${ }^{11}$ Section for Periodontology, Department of Dentistry and Oral Health, Faculty of Health, Aarhus University, Aarhus, Denmark
}

\section{Correspondence}

Ellen Frandsen Lau, Section of Periodontology, Department of Dentistry and Oral Health, Vennelyst Boulevard 9, 8000 Aarhus C, Denmark.

Email: ellen.frandsen@dent.au.dk

Funding information

This study was supported by the World Workshop on Oral Medicine (WWOM) VII.

\begin{abstract}
Objective: The World Workshop on Oral Medicine VII chose the oral microbiome as a focus area. Part 1 presents the methodological state of the science for oral microbiome studies. Part 2 was guided by the question: What is currently known about the microbiome associated with oral squamous cell carcinoma and potentially malignant disorders of the oral mucosa?
\end{abstract}

Materials and Methods: A scoping review methodology was followed to identify and analyse relevant studies on the composition and potential functions of the oral microbiota using high-throughput sequencing techniques. The authors performed searches in PubMed and EMBASE. After removal of duplicates, a total of 239 potentially studies were identified.

Results: Twenty-three studies on oral squamous cell carcinoma, two on oral leukoplakia and four on oral lichen planus were included with substantial differences in diagnostic criteria, sample type, region sequenced and sequencing method utilised. The majority of studies focused on bacterial identification and recorded statistically significant differences in the oral microbiota associated with health and disease. However, even when comparing studies of similar methodology, the microbial 
differences between health and disease varied considerably. No consensus on the composition of the microbiomes associated with these conditions on genus and species level could be obtained. Six studies on oral squamous cell carcinoma had included in silico predicted microbial functions (genes and/or pathways) and found some similarities between the studies.

Conclusions: Attempts to reveal the microbiome associated with oral mucosal diseases are still in its infancy, and the studies demonstrate significant clinical and methodological heterogeneity across disease categories. The immense richness and diversity of the microbiota clearly illustrate that there is a need for additional methodologically comparable studies utilising deep sequencing approaches in significant cohorts of subjects together with functional analyses. Our hope is that following the recipe as outlined in our preceding companion paper, that is Part 1 , will enhance achieving this in the future and elucidate the role of the oral microbiome in oral squamous cell carcinoma and potentially malignant disorders of the oral mucosa.

\section{KEYWORDS}

head and neck cancer, oral microbiome, oral squamous cell carcinoma, potentially malignant disorders of the oral mucosa

\section{1 | INTRODUCTION}

In 2018, 354,864 new oral cancer cases and 177,384 associated deaths were estimated to occur worldwide (Bray et al., 2018). Despite efforts in prevention, early detection and treatment, oral cancer mortality has increased nearly $20 \%$ in the past decade and is expected to continue to rise (Smittenaar, Petersen, Stewart, \& Moitt, 2016). The major known risk factors for oral squamous cell carcinoma (OSCC), which accounts for approximately $85 \%$ of all oral cancers, include tobacco use, heavy alcohol consumption, betel quid chewing and other forms of areca nut usage, and high-risk human papillomavirus (HPV) infection (Conway, Purkayastha, \& Chestnutt, 2018). However, the remaining $15 \%$ of the oral cancers occur without the presence of any of these risk factors (Chocolatewala, Chaturvedi, \& Desale, 2010). All potential aetiological components, including the microbiome, should be considered in the elucidation of aetiopathogenesis, as well as the search for biomarkers for early diagnosis, screening, staging and additional treatment possibilities in order to reduce the rising oral cancer rates. The potentially malignant disorders of the oral mucosa (OPMDs), that is oral leukoplakia, oral erythroplakia, oral lichen planus, palatal lesions in reverse smokers, actinic cheilitis, oral discoid lupus erythematosus and oral submucous fibrosis, should be included in this endeavour.

The involvement of specific microorganisms in cancer development has been implicated for over a century. Associations between bacteria and human malignancies include Helicobacter pylori in gastric cancer and mucosa-associated lymphoid tissue lymphomas, Chlamydia trachomatis in cervical cancer, Salmonella typhi in gallbladder cancer, and Bacteroides fragilis and Fusobacterium nucleatum in colorectal cancer (Mager, 2006). Viral association with cancer includes HPV in cervical, skin, oropharyngeal and anogenital cancers; human T-cell leukaemia virus in adult T-cell leukaemia; human herpesvirus 8 in Kaposi's sarcoma, primary effusion lymphoma and Castleman's disease; Epstein-Barr virus in Burkitt's lymphoma, nasopharyngeal carcinoma, post-transplant lymphomas and Hodgkin's disease; and hepatitis $B$ and $C$ viruses in hepatocellular carcinoma (Morales-Sanchez \& Fuentes-Panana, 2014).

During the last two decades, the association of specific microorganisms other than HPV has also been considered for OSCC and OPMDs by conventional techniques. Culture studies recorded a higher occurrence of the genera Prevotella, Fusobacterium and Veillonella in OSCC lesions as compared to healthy mucosa (Hooper et al., 2006; Nagy, Sonkodi, Szoke, Nagy, \& Newman, 1998). Molecular techniques targeting selected bacterial species suggest associations with OSCC of Streptococcus anginosus (Morita et al., 2003; Sasaki et al., 2005; Tateda et al., 2000), Capnocytophaga gingivalis, Prevotella melaninogenica, Streptococcus mitis (Mager et al., 2005) and Porphyromonas gingivalis (Katz, Onate, Pauley, Bhattacharyya, \& Cha, 2011). Studies using checkerboard DNA-DNA hybridisation and PCR suggested the involvement of Candida albicans in oral cancer (Alnuaimi, Wiesenfeld, O'Brien-Simpson, Reynolds, \& McCullough, 2015) and Streptococcus, Fusobacterium and Capnocytophaga in oral lichen planus (Bornstein, Hakimi, \& Persson, 2008; Wang et al., 2015). Cultivation studies leave the as-yet-uncultured part of the microbiota undetected and the molecular techniques used above suffer from the focus on selected, specific microorganisms. Highperformance DNA sequencing of the 16S rRNA gene has the potential to detect as-yet-uncultured species and cover the majority of microbial taxa in a sample, provided that deep sequencing is performed with an appropriate combination of primers. 
The aim of this scoping review was to present and critically evaluate the published literature on sequence-based determination of the oral microbiome and its potential functions in association with OSCC and OPMDs. Together with Part 1 of this review, this report is designed to provide encouragement for oral medicine specialists to explore this exciting field.

\section{2 | MATERIALS AND METHODS}

\section{1 | Research question}

This review was guided by the question, "What is currently known about the association between the oral microbiome and OSCC and OPMDs, as determined by non-specific sequence-based identification?"

Identifying the microbiota must be accompanied by investigations on properties of the microorganisms involved, and it is in the combination of these approaches that potential aetiopathogenic candidates can be selected and further tested in hypothesis-driven investigations. Therefore, we employed a scoping review methodology, including studies both on identification and on functional properties in order to map the relevant literature, identify key concepts and gaps in research, and trace a path for future research (Daudt, van Mossel, \& Scott, 2013). The format for this scoping review is based on the framework originally outlined by Arksey and O'Malley (2005) and further refined by Levac, Colquhoun, and O'Brien (2010). Our framework includes the following five key phases: (a) identifying the research question, (b) identifying relevant studies, (c) study selection, (d) data characterisation and (e) data summary and synthesis.

\subsection{Identifying relevant studies}

We included all studies published until October 2018 that aimed to determine the oral microbial composition (bacteriome, mycobiome and virome), as well as metagenomic, transcriptomic, and microbial proteomic and metabolomic studies of the microbiota from patients with OSCC and OPMDs. The oral mucosal diseases included in our search were as follows: OSCC, oral leukoplakia, oral erythroplakia,

TAB LE 1 Electronic search strategy for PubMed

\begin{tabular}{|c|c|}
\hline Search term & Search strategy \\
\hline Microbiome & $\begin{array}{l}\text { ((("Microbiota"[Mesh] OR "Microbial Consortia”[Mesh] OR microbiome[Title/Abstract] OR microbiomes[Title/ } \\
\text { Abstract] OR microbiota[Title/Abstract] OR microbiotas[Title/Abstract] OR bacteriome[Title/Abstract] OR } \\
\text { microbial community[Title/Abstract] OR microbial communities[Title/Abstract] OR bacterial community[Title/ } \\
\text { Abstract] OR bacterial communities[Title/Abstract]) OR ("Metagenome"[Mesh] OR "Metagenomics”[Mesh] } \\
\text { OR metagenome[Title/Abstract] OR metagenomic[Title/Abstract] OR metagenomics[Title/Abstract] OR } \\
\text { metagenomes[Title/Abstract]) OR (metatranscriptome[Title/Abstract] OR metatranscriptomic[Title/Abstract] } \\
\text { OR metatranscriptomics[Title/Abstract]) OR (metabolome[Title/Abstract] OR metabolomic[Title/Abstract] OR } \\
\text { metabolomics[Title/Abstract] OR "Metabolome"[Mesh] OR "Metabolomics”[Mesh]) OR (metaproteome[Title/ } \\
\text { Abstract] OR metaproteomic[Title/Abstract] OR metaproteomics[Title/Abstract) OR ("Mycobiome”[Mesh] OR } \\
\text { mycobiome[Title/Abstract] OR mycobiomes[Title/Abstract] OR mycobiota[Title/Abstract] OR mycobiotas[Title/ } \\
\text { Abstract] OR fungal microbiota[Title/Abstract] OR fungal microbiotas[Title/Abstract] OR fungal microbiome[Title/ } \\
\text { Abstract] OR fungal microbiomes[Title/Abstract] OR fungal community[Title/Abstract] OR fungal } \\
\text { communities[Title/Abstract]))) AND English[lang] }\end{array}$ \\
\hline $\begin{array}{l}\text { Oral squamous Cell } \\
\text { carcinoma }\end{array}$ & $\begin{array}{l}\text { (Oral Squamous Cell Carcinoma OR Oral Squamous Cell Carcinomas OR Oral Cavity Squamous Cell Carcinoma } \\
\text { OR Oral Cavity Squamous Cell Carcinomas OR Oropharyngeal Squamous Cell Carcinoma OR Oropharyngeal } \\
\text { Squamous Cell Carcinomas) AND English[lang] }\end{array}$ \\
\hline Oral verrucous carcinoma & $\begin{array}{l}\text { (oral verrucous carcinoma OR oral verrucous carcinomas OR oropharyngeal verrucous carcinoma OR oropharyngeal } \\
\text { verrucous carcinomas) AND English[lang] }\end{array}$ \\
\hline $\begin{array}{l}\text { Leukoplakia OR } \\
\text { Erythroplakia }\end{array}$ & (leukoplakia OR leukoplasia OR Erythroplakia OR Erythroplasia OR erythroleukoplakia) AND English[lang] \\
\hline Lichen planus & (Lichen Planus) AND English[lang] \\
\hline $\begin{array}{l}\text { Palatal lesions in reverse } \\
\text { smokers OR stomatitis } \\
\text { nicotina }\end{array}$ & ((stomatitis nicotina) OR "reverse smokers" OR "reverse smoking") AND English[lang] \\
\hline Actinic keratosis & $\begin{array}{l}\text { (actinic keratosis OR actinic keratoses OR actinic cheilitis OR Actinic cheilosis OR solar keratosis OR solar cheilitis } \\
\text { OR solar cheilosis) AND English[lang] }\end{array}$ \\
\hline $\begin{array}{l}\text { Discoid Lupus } \\
\text { Erythematosus }\end{array}$ & $\begin{array}{l}\text { ((discoid lupus erythematosus) OR (Chronic "Cutaneous Lupus Erythematosus") OR (Cutaneous Lupus } \\
\text { Erythematosus)) AND (English[lang]) }\end{array}$ \\
\hline Oral submucous fibrosis & (oral submucous fibrosis) AND English[lang] \\
\hline Oral dysplasia & $\begin{array}{l}\text { (oral dysplasia OR oropharyngeal dysplasia OR ((("mouth"[MeSH Terms] OR "mouth"[All Fields] OR "oral”[All } \\
\text { Fields]) AND ("epithelial"[All Fields] AND "dysplasia"[All Fields]) OR "epithelial dysplasia"[All Fields])))) OR } \\
\text { ((("oropharynx"[MeSH Terms] OR "oropharynx"[All Fields] OR "oropharyngeal"[All Fields]) AND ("epithelial"[All } \\
\text { Fields] AND "dysplasia"[All Fields]) OR"epithelial dysplasia"[All Fields]))) AND English[lang] }\end{array}$ \\
\hline
\end{tabular}


oral lichen planus, palatal lesions in reverse smokers, actinic cheilitis, oral discoid lupus erythematosus and oral submucous fibrosis. Oral verrucous carcinoma and oral epithelial dysplasia, a histopathological diagnosis associated with an increased risk of malignant transformation, were also included in our searches. Initial literature searches revealed very few studies, and for this reason, we decided not to exclude papers based on poorly described diagnostic criteria.

Since the purpose of this review was to reveal what is known about associations of the oral microbiome with respect to OSCC and OPMDs based on non-specific sequencing, we excluded studies that only utilised culture methods and those that identified a limited number of species. Likewise, we excluded studies focusing on a single or a few microbial properties as well as metabolomic studies that did not allow differentiation between human and microbial metabolites.

\section{3 | Study selection}

We searched the electronic databases PubMed and EMBASE for peer-reviewed papers using a combination of both MeSH terms (translated into Emtree terms for EMBASE search) and free-text forms (Table 1). Individual search strings were constructed for each of the above-mentioned oral mucosal diseases and for oral epithelial dysplasia, using synonyms and derivatives, except for leukoplakia and erythroplakia that were combined in one search, because preliminary searches retrieved the same papers for both diseases. To focus the searches on the microbiota, the search strings for the mucosal conditions were combined with a microbiome search string using search terms related to identification by non-specific sequencing and microbial properties (Table 1). The term "oral" was not included because comparison between searches with and without this term had shown more papers when "oral" was omitted. The search queries were designed by the research team that included an experienced research librarian. The initial searches were performed in PubMed between July 2017 and January 2018. Adjusted search strings were applied to EMBASE in January 2018. The searches were updated monthly until October 2018. Only studies published in English were considered for inclusion. There was no restriction on date of publication. In addition, citations within key articles were searched if they appeared relevant to the review. References were exported into EndNote ${ }^{\circledR}$ X8 (Thomson Reuters), and duplicate citations were removed manually. Three independent reviewers (JR-S, DPB-A and EFL) evaluated titles and abstracts based on eligibility criteria. Reviewers were not blinded to author or journal name. Discordance regarding selection was resolved by discussion after full-text assessment until consensus was reached.

\section{4 | Data characterisation}

All citations deemed relevant after title and abstract screening were procured for subsequent review of the full-text article. A form was developed to confirm relevance and to extract study characteristics such as publication year, country, number of subjects, sample type and technology. In addition, for identification studies, genes sequenced, number of clones/reads identified, taxonomic detection level, main microbial results and study limitations were recorded, whereas for properties studies, type of study, main results and study limitations were recorded (Table S1). The included papers were subdivided into "identification studies" for the articles dealing with identification of the microbiota (bacteriome, mycobiome and virome) and "properties studies" for the articles related to metagenomics, transcriptomics, and microbial proteomics and metabolomics. Data from identification studies were compiled into a Microsoft Excel for Mac (Microsoft Corporation) spreadsheet. Data from properties studies were extracted in essay form and incorporated in the results section.

\section{3 | RESULTS}

A total of 327 potentially relevant studies were the result of the database searches, 87 of which were duplicates. One additional paper was identified through other sources. Titles and abstracts were read, which led to exclusion of 181 references. Full-text analysis of 59 papers resulted in a total of 29 papers for this review. The process of study selection was performed according to the PRISMA statement and presented in Figure 1. The literature search was comprehensive and inclusive, yet zero papers were included for verrucous carcinoma, palatal lesions in reverse smokers, actinic cheilitis, oral discoid lupus erythematosus, oral submucous fibrosis and oral epithelial dysplasia. Relevant papers were only retrieved for OSCC, oral leukoplakia and oral lichen planus. All included papers had identified the microbiome; none had investigated microbial properties. However, six papers on OSCC supplemented the 16S rRNA gene-based species identification with in silico (computer-based) analysis where whole-genome sequences of the species identified were retrieved from public databases and annotated genes compared to give a picture of potential properties.

Table 2 presents the characteristics of the studies included. They are grouped according to the oral condition being investigated, and within each disease type, there is a subdivision based on the microorganisms detected and sample type. Number of participants, technology used, clones/filtered reads per sample, detection level and geographic location of the study are also given as they may impact the results in terms of the microorganisms identified (Gupta, Paul, \& Dutta, 2017) (see Part 1). The predominant microbial taxa at genus and species level are presented in Table 3.

\subsection{The microbiota associated with oral squamous cell carcinoma}

Twenty-three non-specific sequence-based identification studies of the microbiota were included, 14 of which were published in 20172018 (Table 2) (Al-Hebshi, Nasher, Idris, \& Chen, 2015; Al-Hebshi et al., 2017; Bebek et al., 2012; Börnigen et al., 2017; GuerreroPreston et al., 2016, 2017; Hayes et al., 2018; Hooper et al., 2007; Hu, Zhang, Hua, \& Chen, 2016; Lee et al., 2017; Li et al., 2015; Mukherjee et al., 2017; Perera et al., 2017, 2018; Pushalkar et al., 


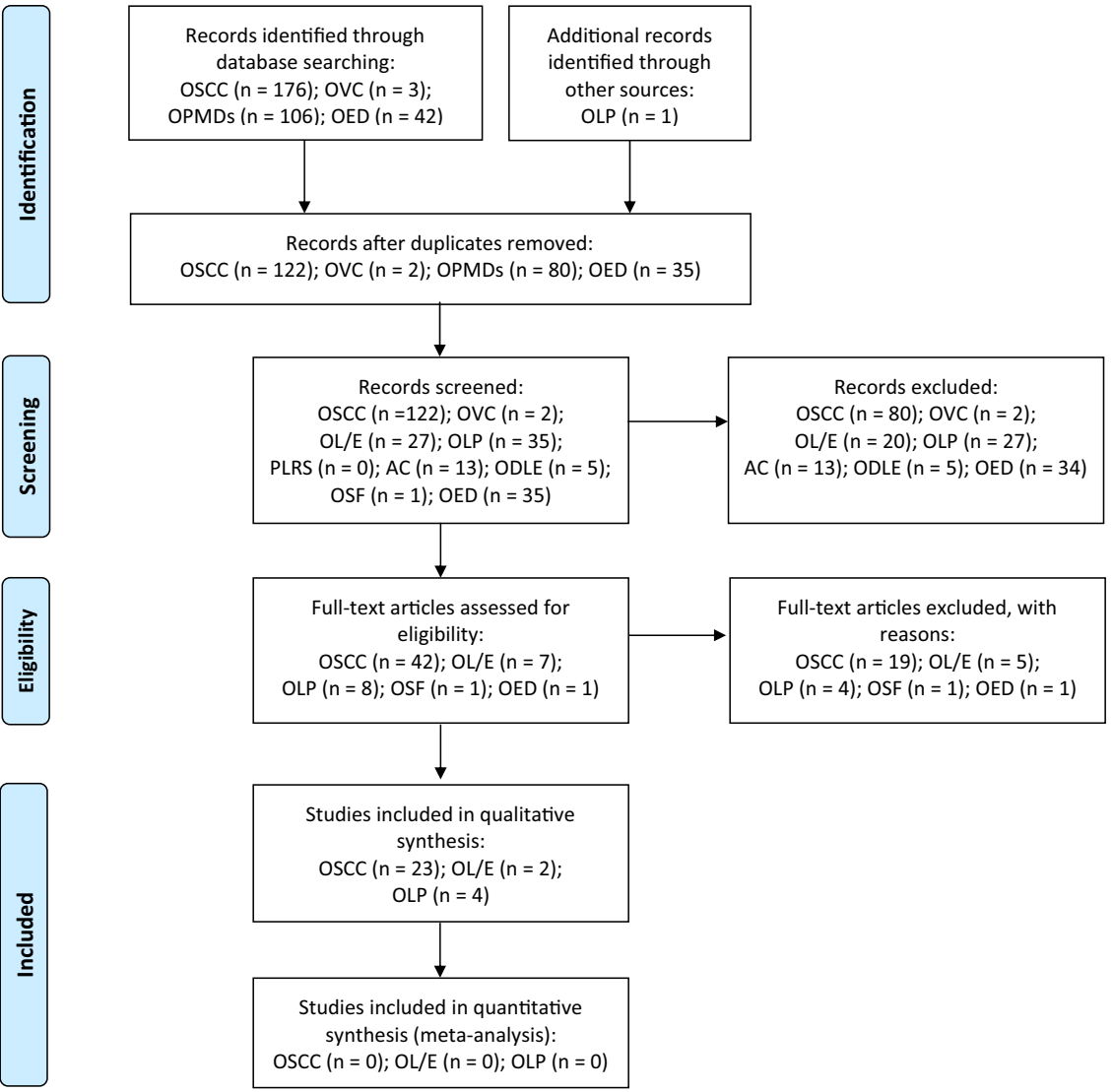

FIGURE 1 Flow diagram showing scoping review synthesis, in accordance with PRISMA guidelines. AC, actinic cheilitis; ODLE, oral discoid lupus erythematosus; OED, oral epithelial dysplasia; OL/E, oral leukoplakia/ erythroplakia; OLP, oral lichen planus; OPMDs, potentially malignant disorders of the oral mucosa; OSCC, oral squamous cell carcinoma; OSF, oral submucous fibrosis; OVC, verrucous carcinoma; PLRS, palatal lesions in reverse smokers
2011, 2012; Schmidt et al., 2014; Shin et al., 2017; Wang et al., 2017; Wolf et al., 2017;Yang, Huang et al., 2018; Yang, Yeh et al., 2018; Zhao et al., 2017). Twenty-one studies focused on bacterial identification (all by $16 \mathrm{~S}$ rRNA gene regions), one of which also identified fungi (ITS2 gene fragment), one only fungi, and one was restricted to viral identification (human rRNA detection followed by search for viral sequences).

The 21 studies identifying the bacteriome consisted of 14 on OSCC exclusively and seven investigating OSCC as part of head and neck squamous cell carcinoma (HNSCC). The latter studies therefore also reported from sample sites outside the oral cavity where the resident, healthy microbiota is different. A variety of sample types (tissue, swabs, saliva and oral rinse/mouthwash samples) was used for both HNSCC and OSCC studies. Investigations utilising tissue samples identified invaded as well as surface-associated microorganisms, whereas investigations utilising oral swab samples identified solely surface-associated microorganisms. In these studies, the control most often was from a contiguous healthy site or an anatomically matched healthy site of the same patient. In studies utilising specimens from saliva and oral rinse/mouthwash, in which the sample represents the entire oral microbial community, the control came from a healthy individual. The studies were divided into two groups based on similar sample types: tissue/swab samples in one group and saliva/oral rinse/mouthwash samples in another group. The number of patients in the bacteriome studies varied considerably, ranging from 3 to 197. Four studies utilised the Sanger sequencing technique, while second-generation sequencing technologies were used by the remaining studies. Various variable regions of the $16 \mathrm{~S}$ rRNA gene were sequenced, ranging from V1 to V5. The number of clones/filtered reads was also highly different; the studies based on Sanger sequencing identified a very limited number of clones due to methodological limitations, whereas those using second-generation sequencing ranged from a few thousand to over 100,000 filtered reads per sample. Studies including a large number of subjects and clones/filtered reads per sample tended to report a higher number of significant differences in the microbiome between health and disease as compared to those studies including a few subjects and clones/filtered reads per sample (Table 3). All studies but one (Bebek et al., 2012) could identify to genus level and many to species level. Finally, the studies had been performed in geographically widespread locations.

In the clear majority of bacteriome studies, statistically significant differences were found between health and disease (Table 3). The reader is referred to the individual papers for detailed information on the statistics employed and to the guidelines on statistical analysis of microbial composition data outlined by Odintsova, Tyakht, and Alexeev (2017).

Of the studies using tissue/swab samples, the one from Bebek et al. (2012) was omitted from the comparison, as reads were identified to phylum and family level only. At the genus level, Parvimonas was found to be significantly increased in tissue/swab samples in the study by Wang et al. (2017) but not by Shin et al. (2017) (Table 3). Of the studies examining saliva/oral rinse/mouthwash samples, Hayes et al. (2018) found no significant 


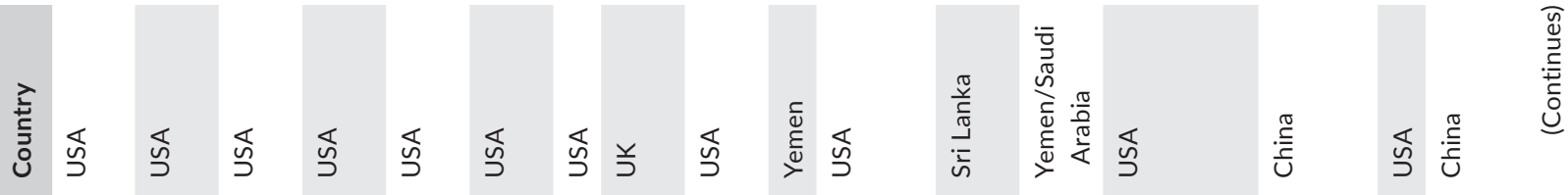

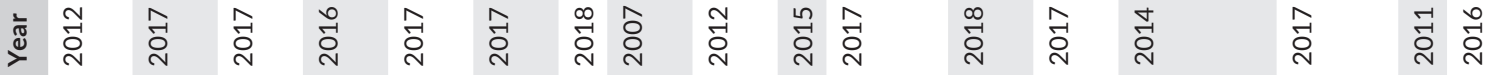

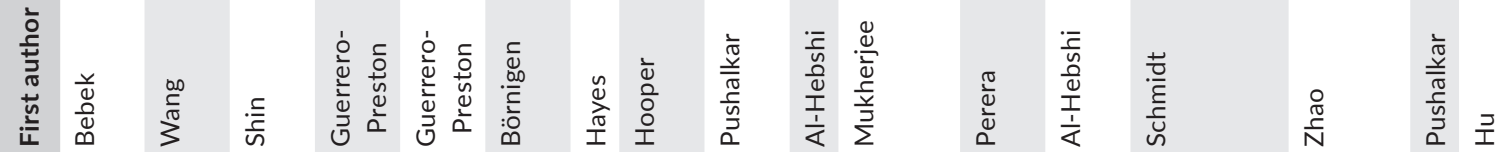

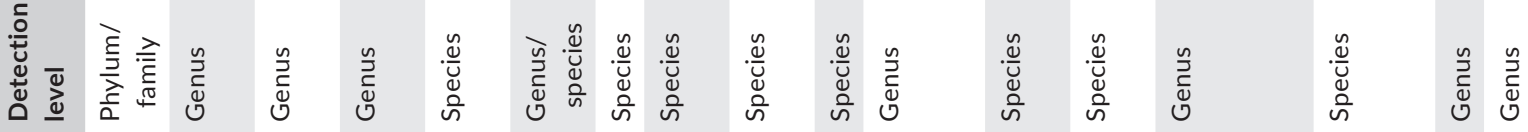

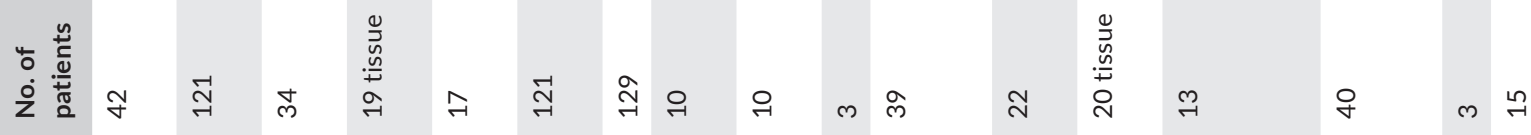

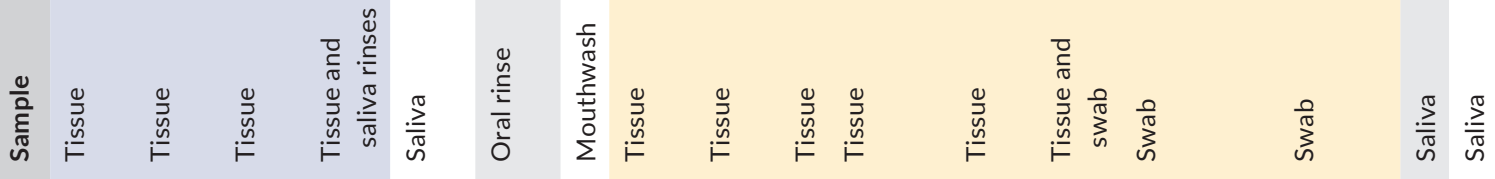

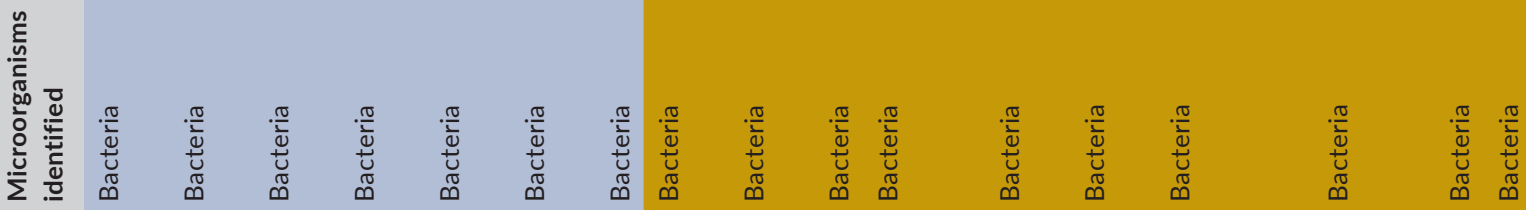

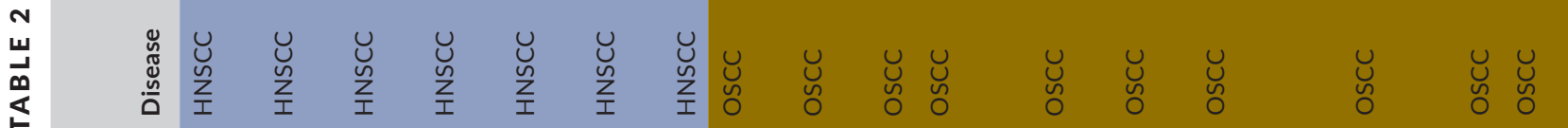




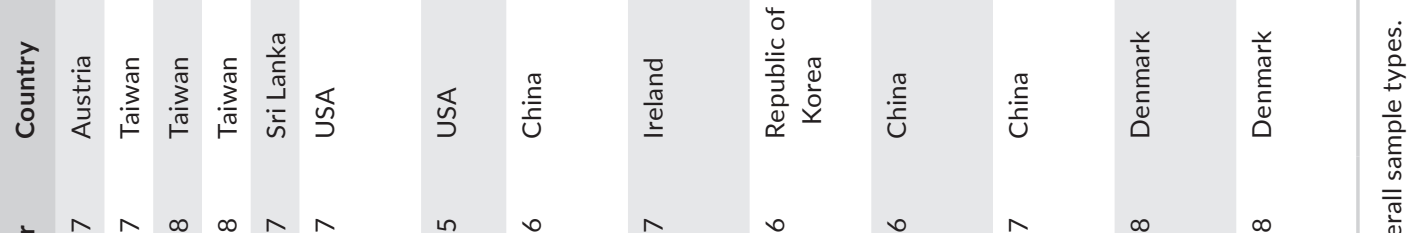

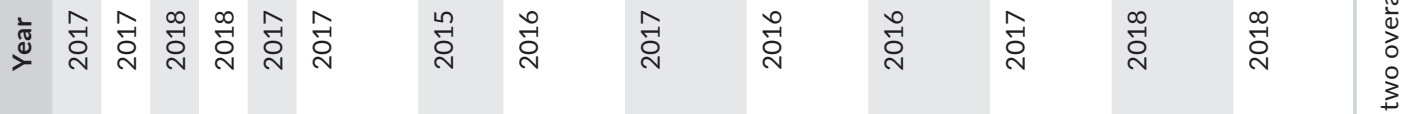

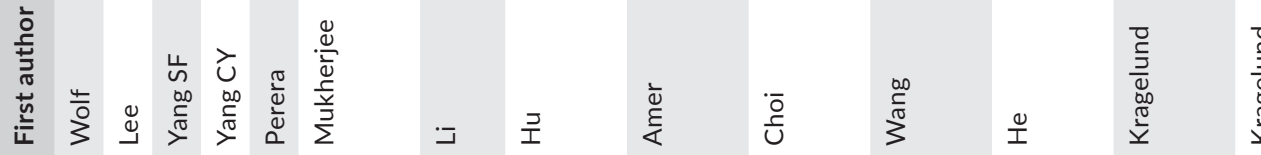

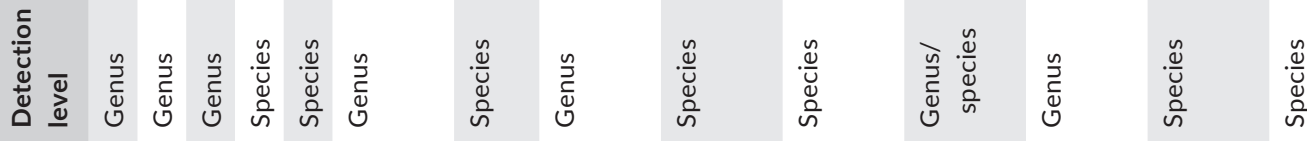

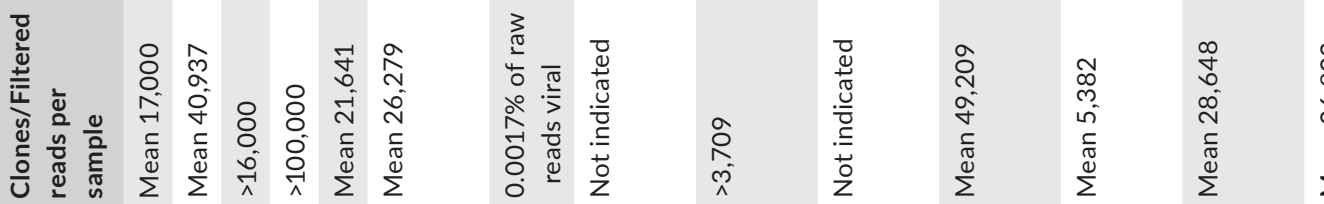

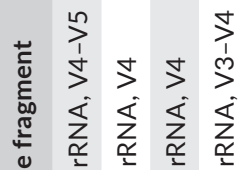

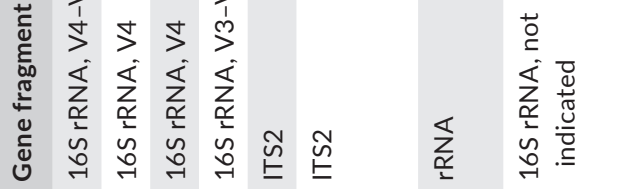

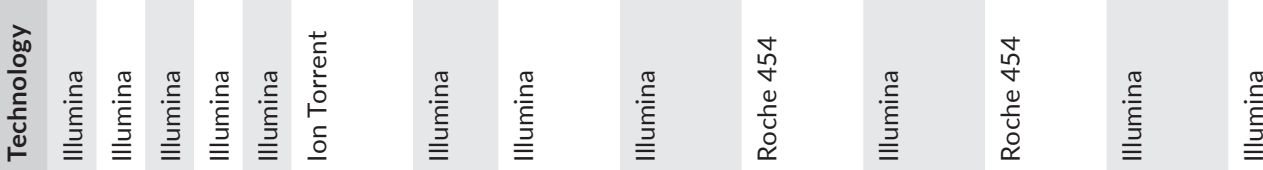

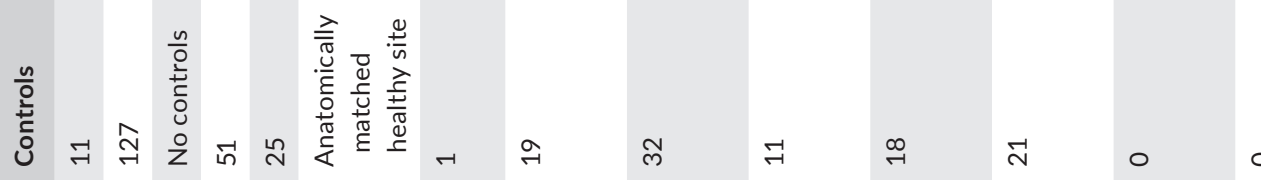

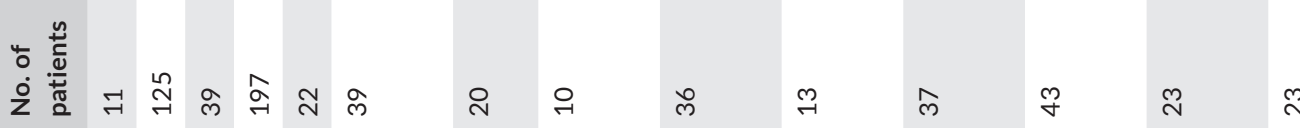

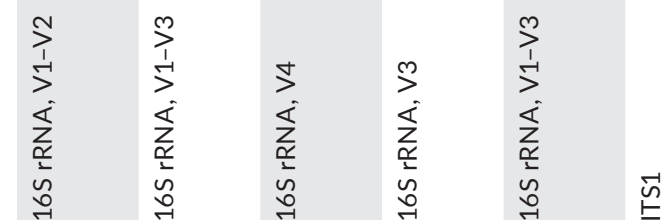

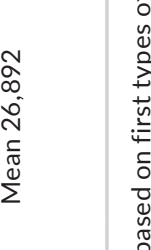




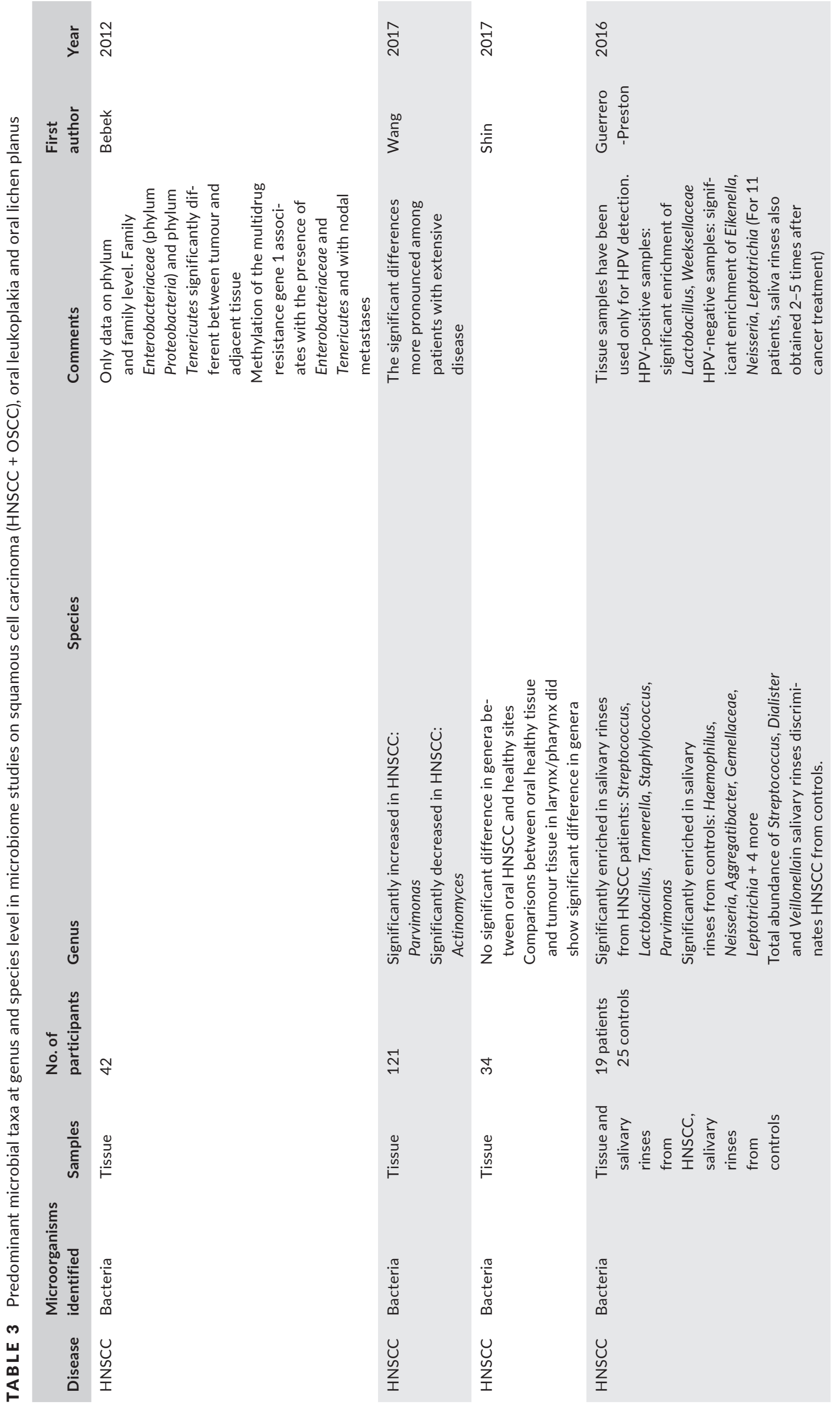




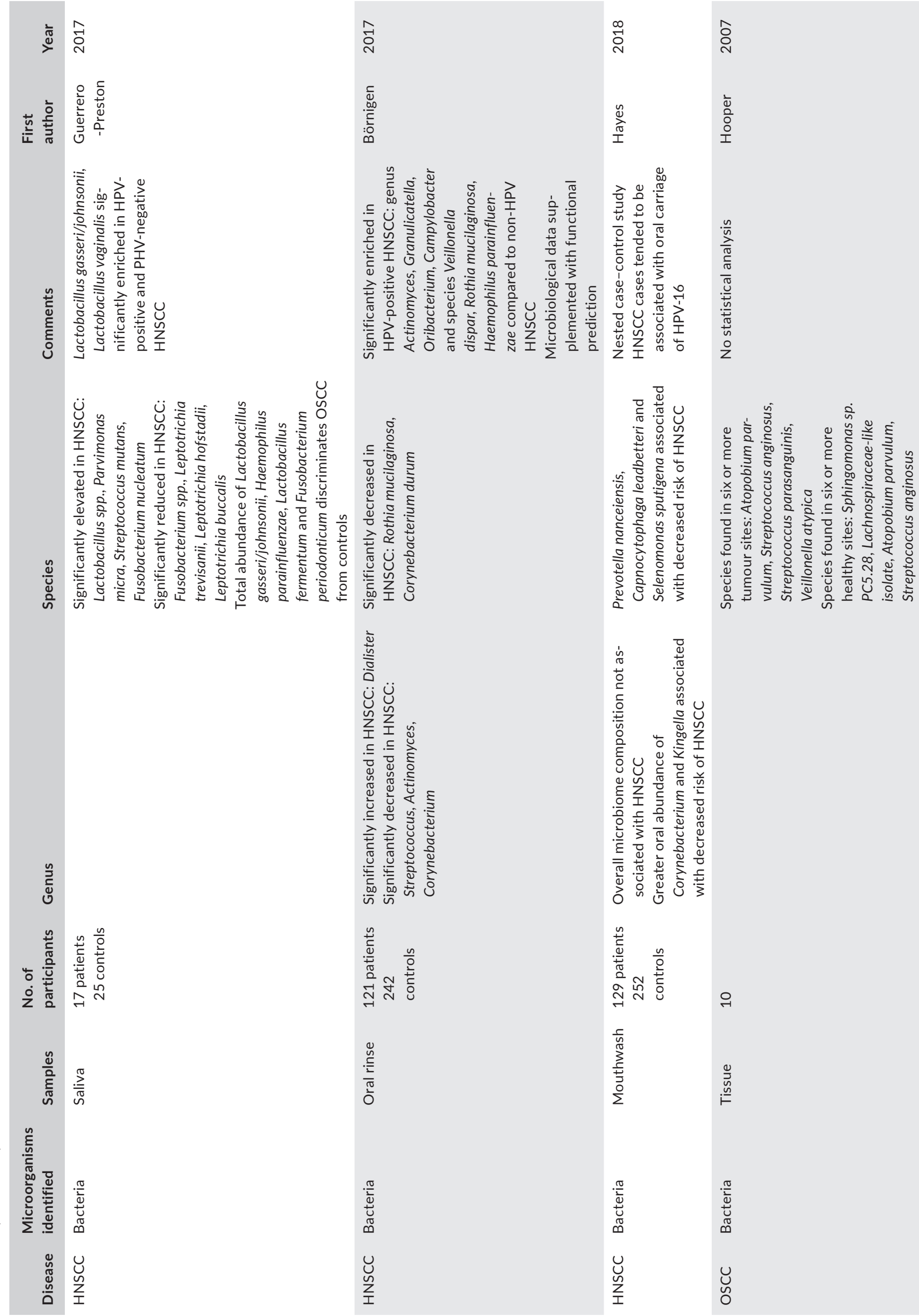



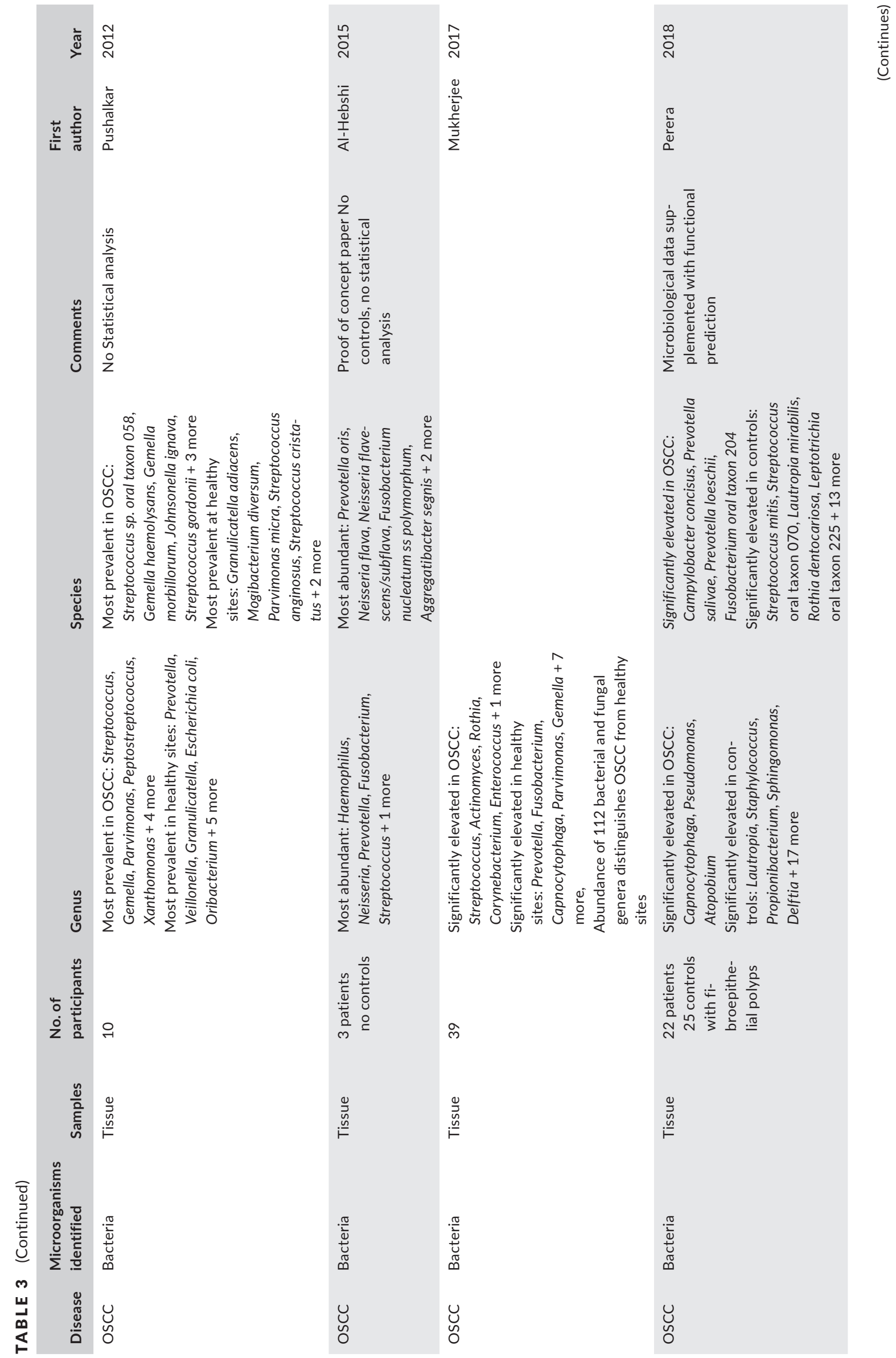


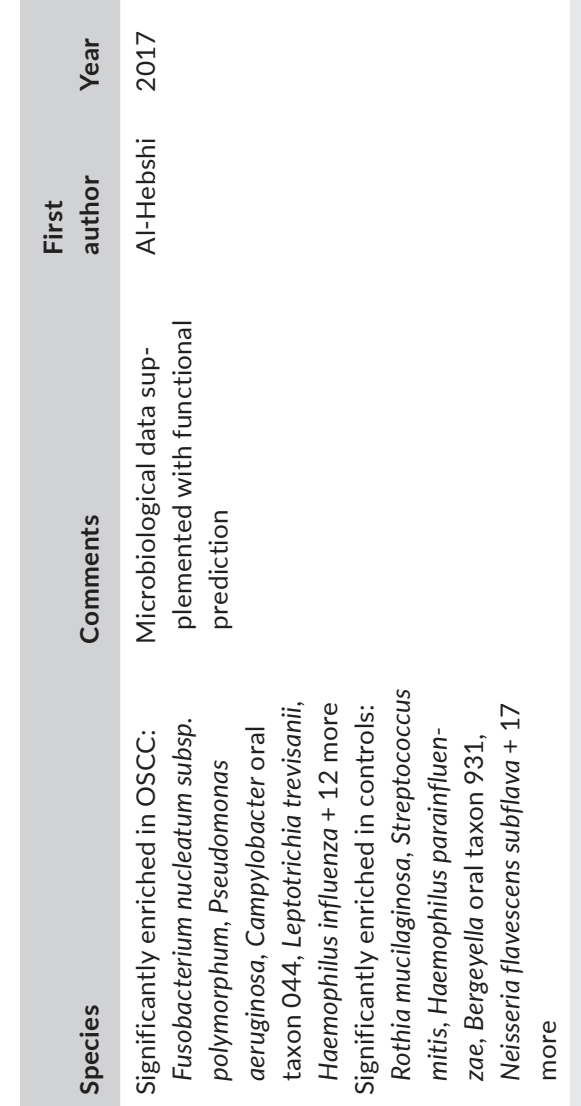

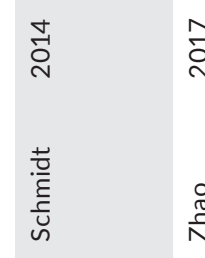

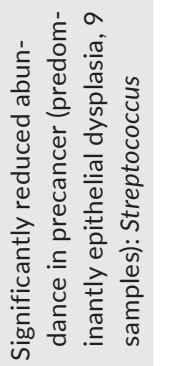

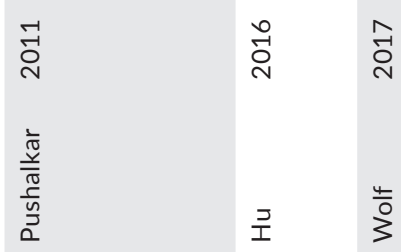

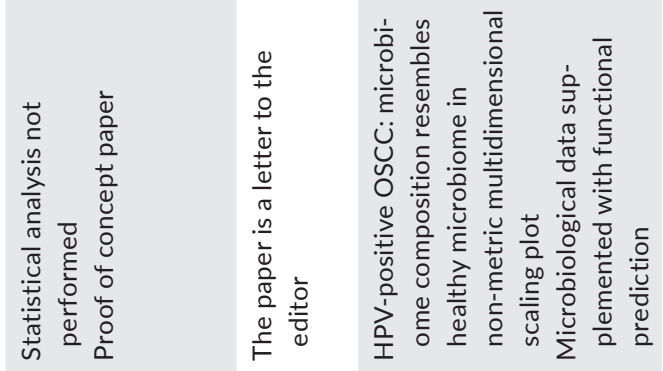

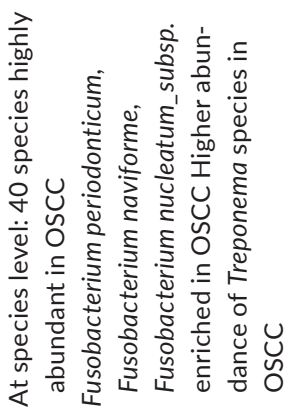
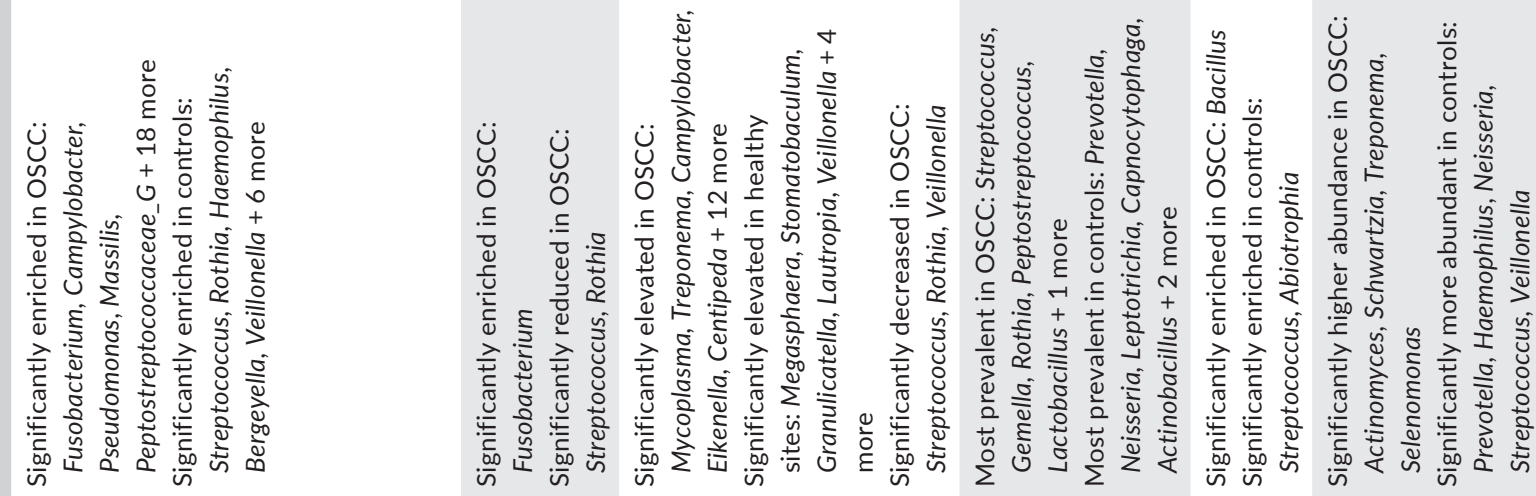

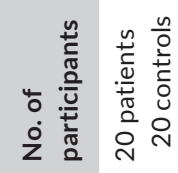
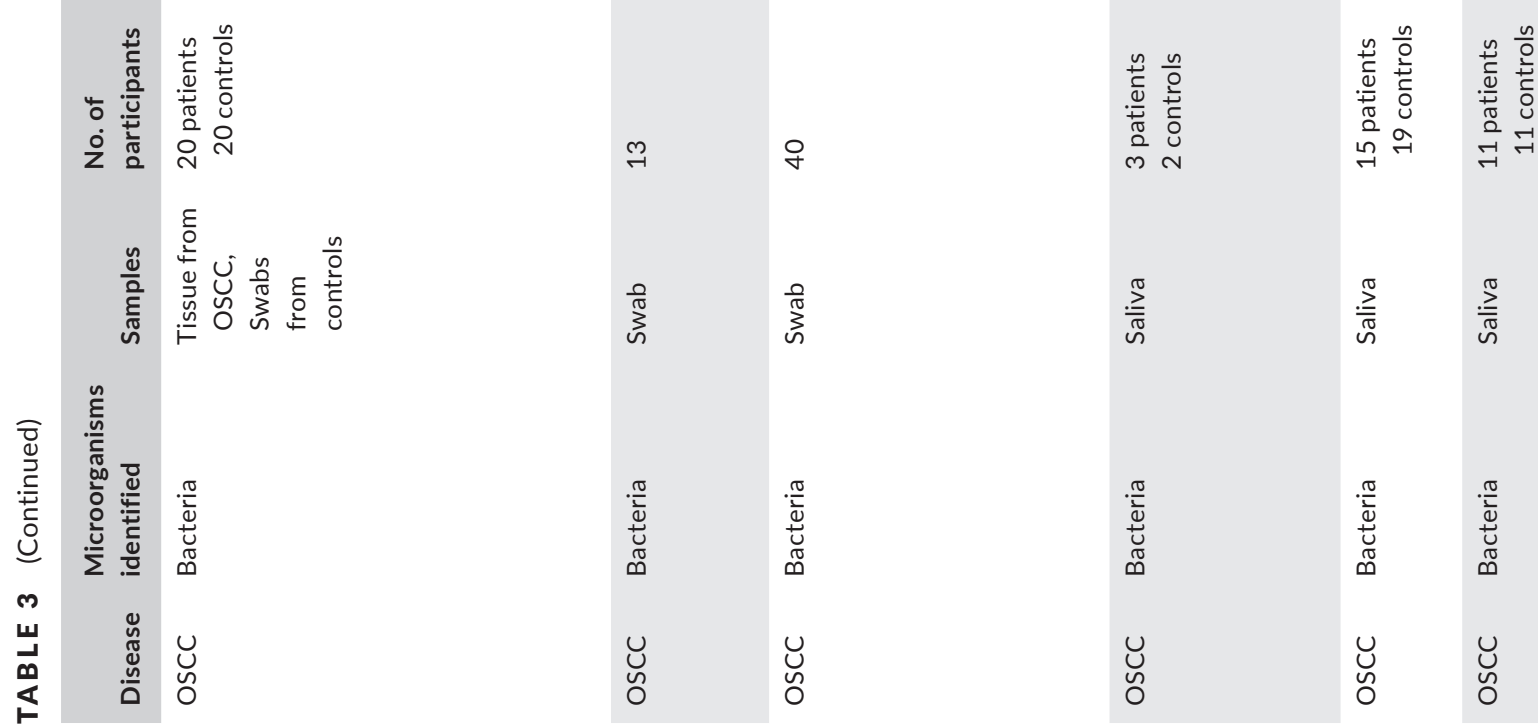

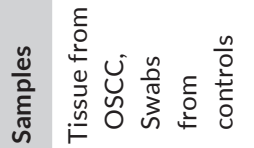


旁

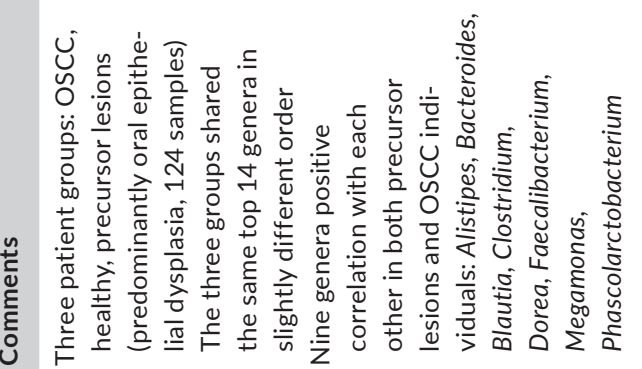

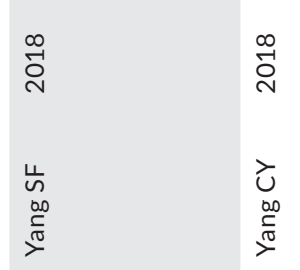

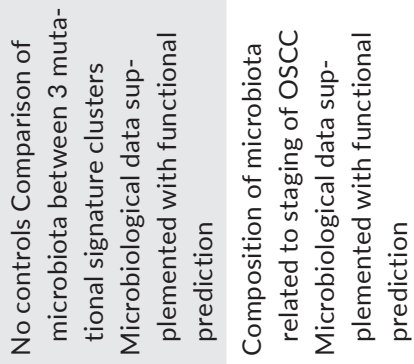
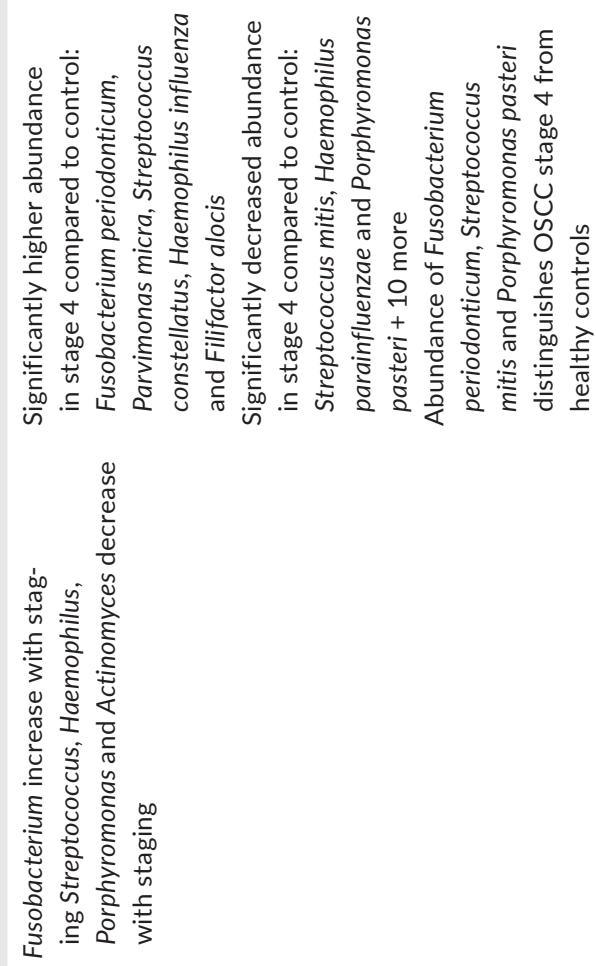

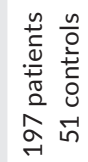

递

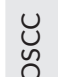

ư 

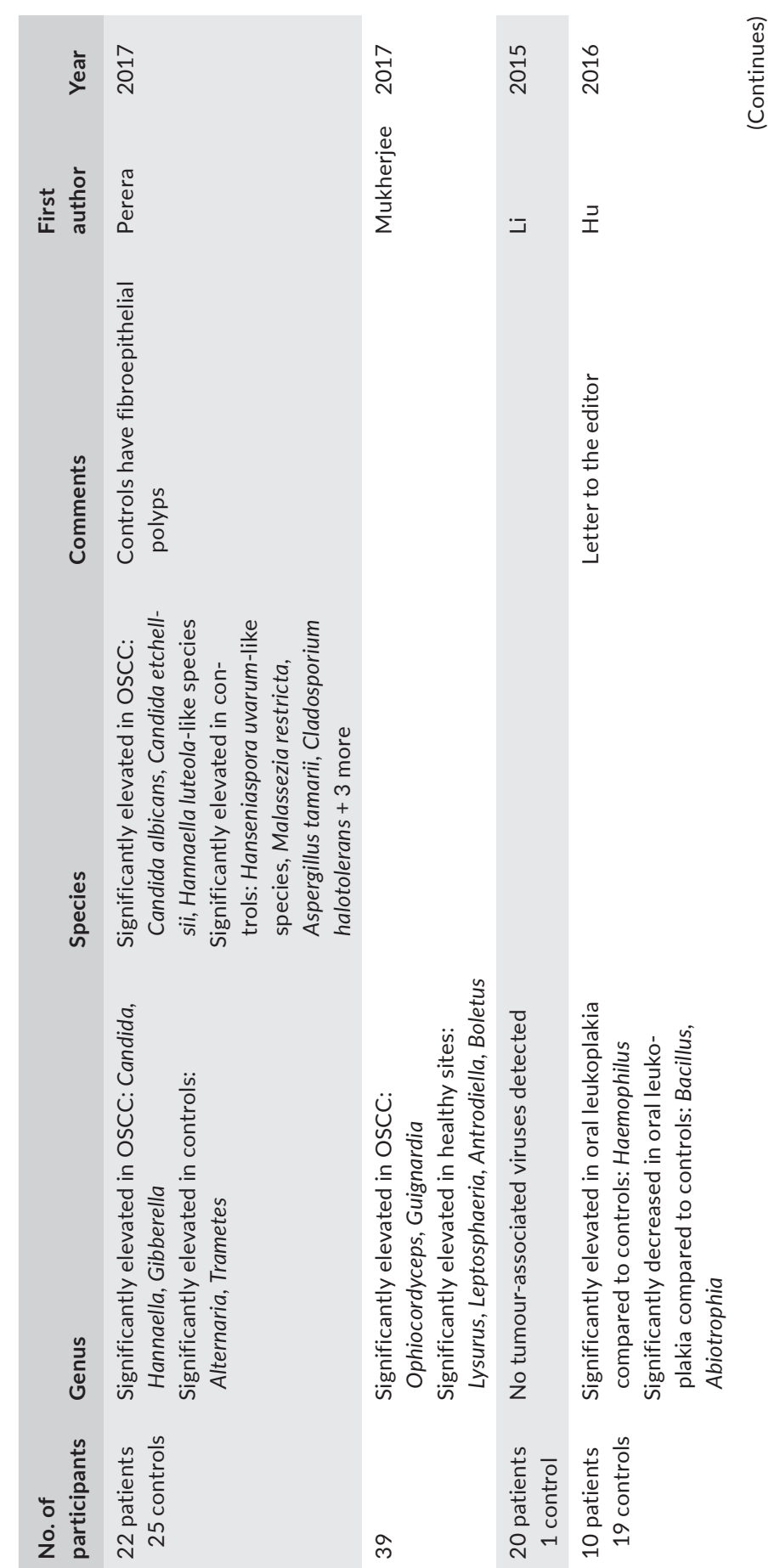

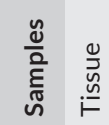
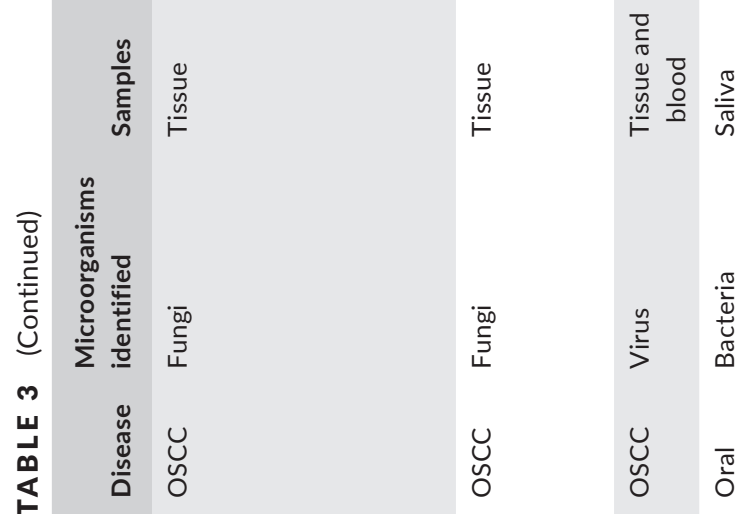

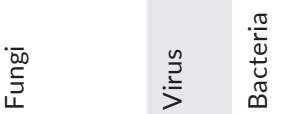

Uू 

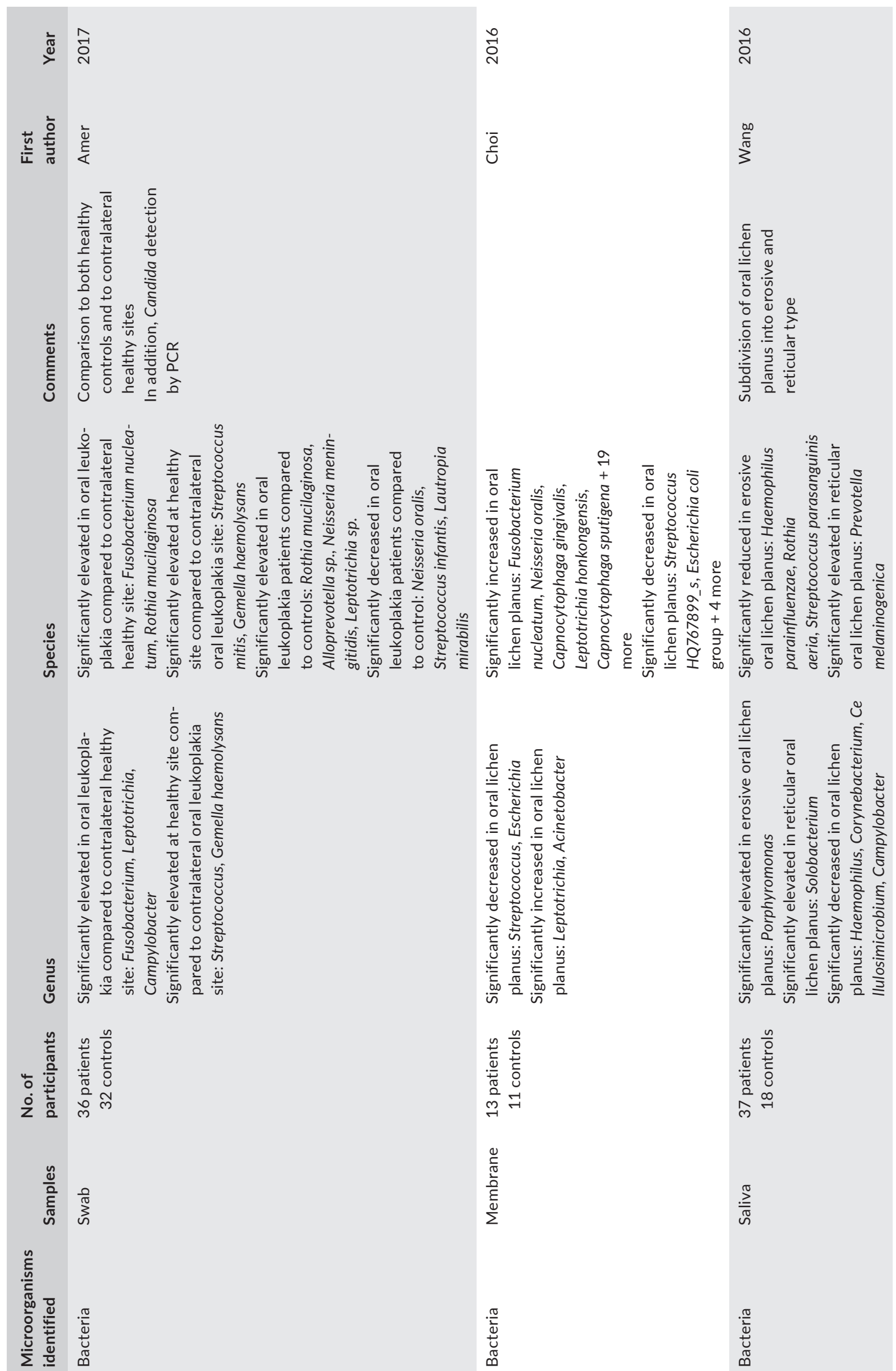

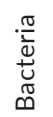

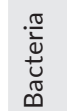

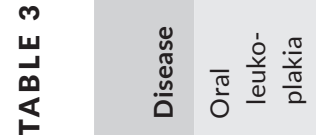

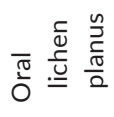

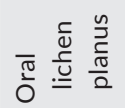


differences at the genus level between health and disease. In contrast, Guerrero-Preston et al. (2016) reported that five genera, that is Streptococcus, Lactobacillus, Tannerella, Staphylococcus and Parvimonas, were significantly enriched in saliva samples from HNSCC patients whereas Börnigen et al. (2017) noted Dialister to be significantly increased (Table 3). On the species level, Lactobacillus sp., Parvimonas micra, Streptococcus mutans and Fusobacterium nucleatum were significantly elevated in HNSCC in a later study by Guerrero-Preston et al. (2017) (Table 3).

The 14 studies that aimed to identify the bacterial part of the microbiome in OSCC were also divided into two groups based on sample type: tissue/swab samples in one group and saliva/oral rinse/mouthwash samples in another group. The genera that were significantly increased in OSCC tissue/swab samples ranged in number from 3 to 23 (Table 3). Among the most abundant genera, no single genus was significantly enriched in all studies involving patients with OSCC. Pushalkar et al. (2012) and Mukherjee et al. (2017) found that Streptococcus was significantly enriched in OSCC, whereas Al-Hebshi et al. (2017) found this genus to be significantly enriched in the controls. Al-Hebshi et al. (2017) and Schmidt et al. (2014) found that Fusobacterium was significantly enriched in OSCC, whereas Mukherjee et al. (2017) found this genus to be significantly enriched in the controls. Three studies reported that Peptostreptococcus was significantly enriched in OSCC (Al-Hebshi et al., 2017; Pushalkar et al., 2012; Zhao et al., 2017). Six studies that analysed tissue/swab samples included identification to species level, in which cases the diversity was even more pronounced. Among the most abundant species in these studies, Fusobacterium nucleatum was the only species that was reported to be significantly enriched in OSCC in more than one study (AIHebshi et al., 2015, 2017; Zhao et al., 2017). All other species were only found to be enriched in a single study. Comparisons of saliva samples showed similar results.

Only two mycobiome studies of OSCC, both using tissue samples, were retrieved (Mukherjee et al., 2017; Perera et al., 2017). Perera et al. (2017) reported three fungal species to be significantly elevated in OSCC compared to control patients with fibroepithelial polyps. Mukherjee et al. (2017), who detected to genus level only, found two genera enriched in OSCC compared to healthy sites. None of these genera were shared with the genera reported by Perera et al. (2017).

The single virome study found no virus to be specifically associated with tongue squamous cell carcinoma samples, including highrisk HPV (Li et al., 2015).

Three studies reported specific combinations of genera/species that differentiated health from disease. At the genus level, Guerrero-Preston et al. (2016) reported that the total abundance of Streptococcus, Dialister and Veillonella discriminated patients with HNSCC from controls, and Lee et al. (2017) found that the abundance of Bacillus, Enterococcus, Parvimonas, Peptostreptococcus and Slackia discriminated saliva samples of patients with OSCC from controls. At the species level, Yang, Yeh et al. (2018) found that the abundance of Fusobacterium periodonticum, Streptococcus 
mitis and Porphyromonas pasteri distinguished swab samples of patients with stage 4 OSCC from controls.

\subsection{The microbiota associated with potentially malignant oral disorders (OPMDs)}

The searches on OPMDs resulted in inclusion of two studies on oral leukoplakia (Amer, Galvin, Healy, \& Moran, 2017; Hu et al., 2016) and four on oral lichen planus (Choi et al., 2016; He et al., 2017; Kragelund \& Keller, 2018; Wang et al., 2016) (Table 2).

The two studies describing the microbiome associated with oral leukoplakia differed in sample type (swab and saliva), which hampers a reliable comparison. The results of these studies differed in the genera found to be associated with OSCC; Haemophilus in the study by Hu et al. (2016) and Fusobacterium, Leptotrichia and Campylobacter in the study by Amer et al. (2017) were found to be significantly enriched in oral leukoplakia (Table 3).

All four studies on oral lichen planus used different sample types for elucidation of the bacteriome. One study (Kragelund \& Keller, 2018) did not include a control group, but described also the mycobiome. No single genus or species among the predominant taxa was significantly elevated in oral lichen planus in two or more of the studies including a control group (Table 3 ).

\section{3 | Potential contribution of the microbiome in oral carcinogenesis}

Six studies identifying the bacterial taxa associated with OSCC by 16S rRNA gene sequencing also predicted the functional properties, mostly with the aid of PICRUSt software (Phylogenetic Investigation of Communities by Reconstruction of Unobserved States). In principle, the programme allocates potential functions based on the species identified and knowledge from whole-genome sequences deposited in public databases. This in silico analysis predicts functions, whereas metagenomic or transcriptomic analyses are necessary for description of the exact functional capabilities of the sample.

Börnigen et al. (2017), Perera et al. (2018) and Al-Hebshi et al. (2017) reported both predicted genes and pathways, whereas Zhao et al. (2017), Yang, Huang et al. (2018), Yang, Yeh et al. (2018) only reported predicted pathways. All studies noted similar differences in the predicted functions between OSCC and health. Three studies reported an increase in genes involved in the synthesis of lipopolysaccharides in oral cancer samples (Al-Hebshi et al., 2017; Börnigen et al., 2017; Perera et al., 2018), while four studies reported an altered amino acid metabolism. Specifically, both Perera et al. (2018) and Al-Hebshi et al. (2017) found that the genes associated with the synthesis of three amino acids were decreased, although they were not the same amino acids. Zhao et al. (2017) found that pathways related to amino acid metabolism were significantly decreased in OSCC, while Yang, Yeh et al. (2018) reported a decrease in amino acid metabolism with increased OSCC staging. There were also, however, conflicting results: Perera et al. (2018) found that genes encoding membrane molecules were enriched in OSCC tissue samples, whereas Zhao et al. (2017) found that pathways related to membrane transport were decreased in swabs from OSCC. In addition, genes associated with glycolysis/glycogenesis were more abundant in the control groups of two studies (Al-Hebshi et al., 2017; Perera et al., 2018), whereas Yang, Yeh et al. (2018) found carbohydrate-related metabolism to increase with OSCC staging.

\section{4 | DISCUSSION}

Most of the 23 papers on OSCC included in this review were published in 2017-2018, which illustrates the increase in microbiome investigations. However, for verrucous carcinoma, OPMDs and oral epithelial dysplasia, the literature was sparse despite very inclusive search criteria. Only two papers on oral leukoplakia and four on oral lichen planus were included. The included papers dealt exclusively with identification of the microbiome; no studies on metagenomics, transcriptomics, or microbial proteomics or metabolomics were retrieved. There is thus potential for advancing the understanding of the relationship between the oral microbiome and oral mucosal diseases regarding "who is there?" (community profiling), "what can they do?" (metagenomics) and "what are they doing?" (transcriptomics, microbial proteomics and metabolomics). This might lead to disclosure of particular microbial compositions that could serve as diagnostic and prognostic markers of disease. It might also reveal specific combinations involved in the aetiology and/or progression of the disease, which in turn could result in the development of novel, targeted treatments, for example probiotics, anti-enzymatic or anti-metabolic substances.

The use of uniform diagnostic criteria and definitions of the diseases were not considered as an inclusion criterion due to the few studies identified in pilot searches. Although the tissue samples analysed in the included studies were histopathologically verified, the diagnostic criteria for HNSCC/OSCC and leukoplakia were poorly described. In the case of oral lichen planus, the majority of the studies used the diagnostic criteria outlined by the WHO (Kramer, Lucas, Pindborg, \& Sobin, 1978). This is a potential source of variations in microbiological results alongside the methodological variables related to the microbiological analyses.

The OSCC studies clearly demonstrate the influence of the many methodological variables on the identified microbiome, as discussed in Part 1 of this review. Pilot literature searches had not revealed many papers, and for that reason, the search permitted the inclusion of OSCC as subtype of HNSCC. Pooling samples from the oral cavity, pharynx and larynx was probably done on the assumption of common biomarkers and/or disease-associated microbiotas, but it has an inherent risk of disclosing significant differences between health and disease that are biologically insignificant due to the diverse sample sites. It is therefore not surprising that the results on HNSCC differed considerably between studies. While the literature search was ongoing, several papers exclusively on OSCC were published. However, the diverse sample types reduced the number of studies relevant for comparison. Saliva and 
tissue samples should not be compared against each other for obvious reasons, and they are also taken for different purposes. Saliva samples are ideal for searching for biomarkers as opposed to elucidating the aetiology of disease where tissue and swab samples are best utilised in disclosing microorganisms that play a potential aetiopathogenic or contributory role. As stated in Part 1 of this review, it is mandatory to consider the sampling strategy both when designing a study and when comparing results. Different sampling methods will inevitably alter the results and hinder comparison (Kragelund \& Keller, 2018).

The introduction of sequencing by universal primers directed at the 16S rRNA gene made it possible to detect as-yet-uncultured species. Although accurate in bacterial identification, Sanger sequencing is labour-intensive, limiting the number of samples and/or clones that can be processed. The 35-96 clones identified per sample in the Sanger OSCC/HNSCC studies (Table 2) are equivalent to the number of colonies picked from an agar plate in culture studies. Apart from the possibility of detecting as-yet-uncultured species, Sanger sequencing thus did not add significantly to the knowledge about the microbiota composition. Studies that used second-generation sequencing reported considerably higher numbers of filtered reads (1,038 to $>100,000$ per sample). The technologies used were Roche 454, Ion Torrent and Illumina. As described in Part 1 (Table S1), Roche 454 , which is no longer supported, produced long reads but at the expense of number of reads per sample. Illumina is now the dominating methodology, characterised by a high number of reads per sample and the possibility of processing many samples in the same run by barcoding (i.e. coding all sequences from the same sample with identical bar sequences). The sequences were initially very short, covering only one variable region of the 16S rRNA gene, allowing identification to genus level. This may provide an overall insight into the potential differences between health and disease. However, species-level or even subspecies-level identification is preferred when seeking aetiopathogenic microorganisms. Recent versions of next-generation sequencing equipment are able to sequence two to three variable regions, which enables identification to species level for most genera. Overall, next-generation sequencing has revealed a much higher number of species in all types of samples than was previously known, and has drawn attention to new taxa. Due to the tremendous species richness within and diversity between samples, it is likely that the differences between health and disease revealed by studies based on a small number of individuals are due to inter-individual variation rather than disease relatedness. Thus, caution must be taken when interpreting the results from microbiome studies.

Four studies on OSCC/HNSCC included over 100 patients and similar numbers of controls (Börnigen et al., 2017; Hayes et al., 2018; Lee et al., 2017; Yang, Yeh et al., 2018). They all tested saliva/mouthwash/oral rinse samples and sequenced either the V3 or the V3-V4 region. Hayes et al. (2018) and Börnigen et al. (2017) investigated patients with HNSCC, and while Hayes et al. (2018) failed to find any significant difference between healthy and diseased sites, Börnigen et al. (2017) reported the genus Dialister to be significantly increased in HNSCC. On the species level, the two studies also differed
(Table 3). Lee et al. (2017) and Yang, Yeh et al. (2018) both tested patients with OSCC patients, but no genera were shared among those significantly enriched in disease (Table 3 ). Despite extended comparability, no uniform picture of the oral microbiota could be drawn from these four large-scale studies.

The two studies on the mycobiome associated with OSCC confirmed the picture of divergent results. Although it is well known that infection with high-risk HPV increases the risk for oral cancer, the only virome study on OSCC did not associate any virus, including high-risk HPV, with tongue squamous cell carcinoma. The same was the case with the studies on the bacteriome associated with oral leukoplakia and oral lichen planus (Table 3).

Collectively, the microbiome studies demonstrate a highly complex diversity in the oral microbiome associated with oral mucosal diseases. Variations in single species most often cannot discriminate between health and disease for those conditions that involve the resident microbiota. Therefore, comparisons of complexes of microorganisms or community-level comparisons are now being included in the analysis. By analogy to periodontal disease, the contribution of the resident oral microbiota to aetiopathogenesis represents a concerted action of many species in a dysbiotic microbiota; however, even discriminative complexes of bacterial taxa differ from one study to another (Table 3). This indicates that identification of the microbiota alone is not enough to reveal the significance of the resident microbiota in relation to oral mucosal diseases. The in silico analyses of the functional properties of the OSCC-associated microbiota revealed by $16 \mathrm{~S}$ rRNA gene sequencing did, in fact, report some similarities especially related to pro-inflammatory properties (Al-Hebshi et al., 2017; Börnigen et al., 2017; Perera et al., 2018). Thus, despite discrepancies in the microbiota, functional similarities in the microbiome associated with OSCC could be found. Likewise, a study using metatranscriptomics reported that although there was highly variable metabolic gene expression of the individual species, the metabolism of the entire microbial community associated with periodontal disease was similar among periodontitis patients (Jorth et al., 2014). In addition to sequence-based identification of the microbiota, metagenomics and transcriptomics revealing what the microorganisms can and are doing have to be included in the elucidation of significant aetiopathogenic microbial combinations.

The very diverse results obtained so far might seem discouraging for the oral medicine specialist who has considered including microbiome analysis to better understand the pathogenesis of oral mucosal diseases, such as OSCC and OPMDs. However, results from microbiome studies have led to effective new treatments of other chronic inflammatory diseases. A short review of the achievements in improved therapy of ulcerative colitis may serve to illustrate this. Ulcerative colitis is a well-described clinical disease with an ill-defined aetiology and remains very challenging to treat. Once triggered in genetically susceptible individuals, an impaired immune response develops, leading to a lack of tolerance to the resident gut microbiota (Lucas Lopez, Grande Burgos, Galvez, \& Perez Pulido, 2017; Thompson-Chagoyan, Maldonado, \& Gil, 2005; Zhang et al., 2017). The gut bacteriome is not yet clearly defined, and neither is 
the mycobiome or virome (Clavel, Lagkouvardos, \& Hiergeist, 2016; Gupta et al., 2017; Nash et al., 2017; Virgin, 2014; Zhang et al., 2017). Nevertheless, the microbial differences between health and disease have led to experimental treatment protocols involving probiotics and faecal transplantation. A number of systematic reviews conclude that faecal microbiota transplantation induces remission in some patients with active ulcerative colitis (Costello et al., 2017; Paramsothy et al., 2017). The results on probiotics are more equivocal (Ghouri et al., 2014), maybe due to random choice of species. However, a recent systematic review highlighted that three trials using the same combination of probiotics, tested on a total of 319 patients, caused remission in active ulcerative colitis (Derwa, Gracie, Hamlin, \& Ford, 2017). So, despite an incomplete understanding of the healthy gut microbiome and the pathogenesis of ulcerative colitis, microbiome studies have enabled researchers to formulate and test hypotheses that have produced promising interventions. The same approach has been tested to a limited extent for its potential to influence gingival health and reduce counts of Streptococcus mutans (Seminario-Amez, Lopez-Lopez, Estrugo-Devesa, Ayuso-Montero, \& Jane-Salas, 2017) and Candida (Keller \& Kragelund, 2018) with variable results.

The oral medicine specialist is therefore encouraged to enter the field of microbiome research. There is both ample opportunity and a clear need for oral medicine specialists who have access to valuable study populations to collaborate with researchers in microbiology, gene sequencing, bioinformatics and biostatistics, to unravel the potential contribution of the oral microbiota as a predictor and aetiological factor in oral mucosal diseases. This review illustrates the difficulties in comparing studies using diverse methodologies. In order to draw solid conclusions, more studies using the same methodology as well as multicentre studies are preferred, as they may inform on the influence of ethnic and environmental variations. It has been repeatedly mentioned that association studies do not indicate aetiological significance of the microorganisms that differ between health and disease. This is unequivocally true. It is also evident that the choice of methods greatly influences the results. Nevertheless, association studies can be valuable for identifying species or microbial compositions of potential importance that can be further studied for their value as prognostic markers of disease or their aetiological and contributory significance in hypothesis-driven studies. In that respect, the oral medicine specialists are encouraged to establish longitudinal cohort studies, which are ideal, albeit cumbersome, to determine both aetiopathogenic relationships and prognostic markers. A recent study involving two US cancer cohorts showed that enrichment and depletion of specific oral bacterial species in mouthwash samples taken before the cancer diagnosis were associated with higher risk of oesophageal cancer development (Peters et al., 2017). Such studies can lead to development of rapid diagnostic tests. The methods are already available, for example the microarray technique (HOMINGS (Human Oral Microbe Identification using Next Generation Sequencing) (Mougeot et al., 2016) and PathoChip (specific cancer-associated species) (Banerjee et al., 2017)). Finally, identification of the microbiome by targeting specific genes cannot stand alone. Metagenomics and transcriptomics adding knowledge of microbial functional properties are crucial methods for an increased understanding of the oral microbial community in health and disease. As pointed out in Part 1, such studies should follow a standardised protocol evolved in collaboration with researchers with microbiological, sequencing, bioinformatics and statistical skills. The contribution of the oral medicine specialist is essential for accurate diagnosis of the patients based on clear diagnostic criteria.

\section{5 | CONCLUSIONS}

High-throughput sequencing has made it possible to determine the microbial composition as well as the functional properties of a sample quickly and at relatively low costs. Of the oral mucosal diseases, OSCC is the only one where it has been applied to any appreciable extent. The studies have not led to a common concept of the microbiota associated with health and disease, probably due to inaccurate diagnosis, numerous methodological variations, a high species richness and an immense diversity between samples. There is clearly a need for more and comparable designed microbiome studies of oral mucosal diseases. As pointed out in Part 1 of this review, such studies should follow a standardised protocol evolved in collaboration with experts in microbiology, sequencing, bioinformatics and biostatistics. The oral medicine specialist is essential for initiation of studies as he/she has access to relevant study populations.

\section{ACKNOWLEDGEMENTS}

We thank Thomas Clavel (University clinic Aachen, Germany) for critical reviewing of the manuscript and fruitful discussions. The WWOM VII Steering Committee gratefully acknowledges the following organizations and companies that provided financial support for WWOM VII: American Academy of Oral Medicine, European Association of Oral Medicine, The British Society for Oral Medicine, Oral Diseases, Henry Schein Cares Foundation, Colgate Palmolive, Xerostom, Afyx, The World Dental Education Foundation, and Unilever. In addition, research reported in this publication was supported by the National Institute Of Dental \& Craniofacial Research of the National Institutes of Health under Award Number R13DE027613. The content is solely the responsibility of the authors and does not necessarily represent the official views of the National Institutes of Health.

\section{CONFLICT OF INTEREST}

None to declare.

\section{AUTHOR CONTRIBUTIONS}

J. Robledo-Sierra, D. Porat Ben-Amy, E. Varoni, R. Bavarian, R. Kerr, D. E. Peterson and E. Frandsen Lau designed the study. J. RobledoSierra, D. Porat Ben-Amy, J. Lytoft Simonsen and E. Frandsen Lau performed the literature search and study analysis. J. Robledo-Sierra, 
D. Porat Ben-Amy and E. Frandsen Lau drafted the manuscript, and all authors participated in critical editing of the manuscript.

\section{ORCID}

Alexander R. Kerr (iD https://orcid.org/0000-0002-2162-8572

Douglas E. Peterson iD https://orcid.org/0000-0002-2665-4964

Ellen Frandsen Lau iD https://orcid.org/0000-0001-8468-3256

\section{REFERENCES}

Al-Hebshi, N. N., Nasher, A. T., Idris, A. M., \& Chen, T. (2015). Robust species taxonomy assignment algorithm for $16 \mathrm{~S}$ rRNA NGS reads: Application to oral carcinoma samples. Journal of Oral Microbiology, 7 , 28934. https://doi.org/10.3402/jom.v7.28934

Al-Hebshi, N. N., Nasher, A. T., Maryoud, M. Y., Homeida, H. E., Chen, T., Idris, A. M., \& Johnson, N. W. (2017). Inflammatory bacteriome featuring Fusobacterium nucleatum and Pseudomonas aeruginosa identified in association with oral squamous cell carcinoma. Scientific Reports, 7, 1834. https://doi.org/10.1038/ s41598-017-02079-3

Alnuaimi, A. D., Wiesenfeld, D., O'Brien-Simpson, N. M., Reynolds, E. C., \& McCullough, M. J. (2015). Oral Candida colonization in oral cancer patients and its relationship with traditional risk factors of oral cancer: A matched case-control study. Oral Oncology, 51, 139-145. https ://doi.org/10.1016/j.oraloncology.2014.11.008

Amer, A., Galvin, S., Healy, C. M., \& Moran, G. P. (2017). The microbiome of potentially malignant oral leukoplakia exhibits enrichment for Fusobacterium, Leptotrichia, Campylobacter, and Rothia Species. Frontiers in Microbiology, 8, 2391. https://doi.org/10.3389/ fmicb.2017.02391

Arksey, H., \& O'Malley, L. (2005). Scoping studies: Towards a methodological framework. International Journal of Social Research Methodology, 8, 19-32. https://doi.org/10.1080/1364557032000119616

Banerjee, S., Tian, T., Wei, Z., Peck, K. N., Shih, N., Chalian, A. A., ... Robertson, E. S. (2017). Microbial signatures associated with oropharyngeal and oral squamous cell carcinomas. Scientific Reports, 7, 4036. https://doi.org/10.1038/s41598-017-03466-6

Bebek, G., Bennett, K. L., Funchain, P., Campbell, R., Seth, R., Scharpf, J., ... Eng, C. (2012). Microbiomic subprofiles and MDR1 promoter methylation in head and neck squamous cell carcinoma. Human Molecular Genetics, 21, 1557-1565. https://doi.org/10.1093/hmg/ddr593

Börnigen, D., Ren, B., Pickard, R., Li, J., Ozer, E., Hartmann, E. M., ... Huttenhower, C. (2017). Alterations in oral bacterial communities are associated with risk factors for oral and oropharyngeal cancer. Scientific Reports, 7, 17686. https://doi.org/10.1038/s41598-017-17795-z

Bornstein, M. M., Hakimi, B., \& Persson, G. R. (2008). Microbiological findings in subjects with asymptomatic oral lichen planus: A crosssectional comparative study. Journal of Periodontology, 79, 23472355. https://doi.org/10.1902/jop.2008.080303

Bray, F., Ferlay, J., Soerjomataram, I., Siegel, R. L., Torre, L. A., \& Jemal, A. (2018). Global cancer statistics 2018: GLOBOCAN estimates of incidence and mortality worldwide for 36 cancers in 185 countries. CA: A Cancer Journal for Clinicians, 68, 394-424. https://doi.org/10.3322/ caac. 21492.

Chocolatewala, N., Chaturvedi, P., \& Desale, R. (2010). The role of bacteria in oral cancer. Indian Journal of Medical and Paediatric Oncology, 31, 126-131. https://doi.org/10.4103/0971-5851.76195

Choi, Y. S., Kim, Y., Yoon, H. J., Baek, K. J., Alam, J., Park, H. K., \& Choi, Y. (2016). The presence of bacteria within tissue provides insights into the pathogenesis of oral lichen planus. Scientific Reports, 6, 1-13. https://doi.org/10.1038/srep29186.

Clavel, T., Lagkouvardos, I., \& Hiergeist, A. (2016). Microbiome sequencing: Challenges and opportunities for molecular medicine. Expert Review of Molecular Diagnostics, 16, 795-805. https://doi. org/10.1080/14737159.2016.1184574

Conway, D. I., Purkayastha, M., \& Chestnutt, I. G. (2018). The changing epidemiology of oral cancer: Definitions, trends, and risk factors. British Dental Journal, 225, 867-873. https://doi.org/10.1038/ sj.bdj.2018.922

Costello, S. P., Soo, W., Bryant, R. V., Jairath, V., Hart, A. L., \& Andrews, J. M. (2017). Systematic review with meta-analysis: Faecal microbiota transplantation for the induction of remission for active ulcerative colitis. Alimentary Pharmacology and Therapeutics, 46, 213-224. https ://doi.org/10.1111/apt.14173

Daudt, H. M., van Mossel, C., \& Scott, S. J. (2013). Enhancing the scoping study methodology: A large, inter-professional team's experience with Arksey and O'Malley's framework. BMC Medical Research Methodology, 13, 48. https://doi.org/10.1186/1471-2288-13-48

Derwa, Y., Gracie, D. J., Hamlin, P. J., \& Ford, A. C. (2017). Systematic review with meta-analysis: The efficacy of probiotics in inflammatory bowel disease. Alimentary Pharmacology and Therapeutics, 46, 389-400. https://doi.org/10.1111/apt.14203

Ghouri, Y. A., Richards, D. M., Rahimi, E. F., Krill, J. T., Jelinek, K. A., \& DuPont, A. W. (2014). Systematic review of randomized controlled trials of probiotics, prebiotics, and synbiotics in inflammatory bowel disease. Clinical and Experimental Gastroenterology, 7, 473-487.

Guerrero-Preston, R., Godoy-Vitorino, F., Jedlicka, A., Rodriguez-Hilario, A., Gonzalez, H., Bondy, J., ... Sidransky, D. (2016). 16S rRNA amplicon sequencing identifies microbiota associated with oral cancer, Human Papilloma Virus infection and surgical treatment. Oncotarget, 7, 51320-51334.

Guerrero-Preston, R., White, J. R., Godoy-Vitorino, F., Rodriguez-Hilario, A., Navarro, K., Gonzalez, H., ... Sidransky, D. (2017). High-resolution microbiome profiling uncovers Fusobacterium nucleatum, Lactobacillus gasseri/johnsonii, and Lactobacillus vaginalis associated to oral and oropharyngeal cancer in saliva from HPV positive and HPV negative patients treated with surgery and chemo-radiation. Oncotarget, 8, 110931-110948.

Gupta, V. K., Paul, S., \& Dutta, C. (2017). Geography, ethnicity or subsistence-specific variations in human microbiome composition and diversity. Frontiers in Microbiology, 8, 1162. https://doi.org/10.3389/ fmicb.2017.01162

Hayes, R. B., Ahn, J., Fan, X., Peters, B. A., Ma, Y., Yang, L., ... Pei, Z. (2018). Association of oral microbiome with risk for incident head and neck squamous cell cancer. JAMA Oncology, 4, 358-365. https:// doi.org/10.1001/jamaoncol.2017.4777

He, Y., Gong, D., Shi, C., Shao, F., Shi, J., \& Fei, J. (2017). Dysbiosis of oral buccal mucosa microbiota in patients with oral lichen planus. Oral Diseases, 23, 674-682. https://doi.org/10.1111/odi.12657

Hooper, S. J., Crean, S. J., Fardy, M. J., Lewis, M. A., Spratt, D. A., Wade, W. G., \& Wilson, M. J. (2007). A molecular analysis of the bacteria present within oral squamous cell carcinoma. Journal of Medical Microbiology, 56, 1651-1659. https://doi.org/10.1099/jmm.0.46918-0

Hooper, S. J., Crean, S. J., Lewis, M. A., Spratt, D. A., Wade, W. G., \& Wilson, M. J. (2006). Viable bacteria present within oral squamous cell carcinoma tissue. Journal of Clinical Microbiology, 44, 1719-1725. https://doi.org/10.1128/JCM.44.5.1719-1725.2006

Hu, X., Zhang, Q., Hua, H., \& Chen, F. (2016). Changes in the salivary microbiota of oral leukoplakia and oral cancer. Oral Oncology, 56, e6-e8. https://doi.org/10.1016/j.oraloncology.2016.03.007

Jorth, P., Turner, K. H., Gumus, P., Nizam, N., Buduneli, N., \& Whiteley, M. (2014). Metatranscriptomics of the human oral microbiome during health and disease. mBio, 5, e01012-e01014. 
Katz, J., Onate, M. D., Pauley, K. M., Bhattacharyya, I., \& Cha, S. (2011). Presence of Porphyromonas gingivalis in gingival squamous cell carcinoma. International Journal of Oral Science, 3, 209-215. https://doi. org/10.4248/IJOS11075

Keller, M. K., \& Kragelund, C. (2018). Randomized pilot study on probiotic effects on recurrent candidiasis in oral lichen planus patients. Oral Diseases, 24, 1107-1114. https://doi.org/10.1111/odi.12858

Kragelund, C., \& Keller, M. K. (2018). The oral microbiome in oral lichen planus during a 1-year randomized clinical trial. Oral Diseases, 25, 327-338.

Kramer, I. R., Lucas, R. B., Pindborg, J. J., \& Sobin, L. H. (1978). Definition of leukoplakia and related lesions: An aid to studies on oral precancer. Oral Surgery, Oral Medicine, Oral Pathology, 46, 518-539.

Lee, W. H., Chen, H. M., Yang, S. F., Liang, C., Peng, C. Y., Lin, F. M., ... Jong, Y. J. (2017). Bacterial alterations in salivary microbiota and their association in oral cancer. Scientific Reports, 7, 16540. https:// doi.org/10.1038/s41598-017-16418-x

Levac, D., Colquhoun, H., \& O'Brien, K. K. (2010). Scoping studies: Advancing the methodology. Implementation Science, 5, 69. https:// doi.org/10.1186/1748-5908-5-69

Li, R., Faden, D. L., Fakhry, C., Langelier, C., Jiao, Y., Wang, Y., ... Agrawal, N. (2015). Clinical, genomic, and metagenomic characterization of oral tongue squamous cell carcinoma in patients who do not smoke. Head and Neck, 37, 1642-1649. https://doi.org/10.1002/hed.23807

Lucas Lopez, R., Grande Burgos, M. J., Galvez, A., \& Perez Pulido, R. (2017). The human gastrointestinal tract and oral microbiota in inflammatory bowel disease: A state of the science review. APMIS, 125, 3-10. https://doi.org/10.1111/apm.12609

Mager, D. L. (2006). Bacteria and cancer: Cause, coincidence or cure? A review Journal of Translational Medicine, 4, 14. https://doi. org/10.1186/1479-5876-4-14

Mager, D. L., Haffajee, A. D., Devlin, P. M., Norris, C. M., Posner, M. R., \& Goodson, J. M. (2005). The salivary microbiota as a diagnostic indicator of oral cancer: A descriptive, non-randomized study of cancer-free and oral squamous cell carcinoma subjects. Journal of Translational Medicine, 3, 27. https://doi. org/10.1186/1479-5876-3-27

Morales-Sanchez, A., \& Fuentes-Panana, E. M. (2014). Human viruses and cancer. Viruses, 6, 4047-4079. https://doi.org/10.3390/v6104047

Morita, E., Narikiyo, M., Yano, A., Nishimura, E., Igaki, H., Sasaki, H., ... Kawabe, R. (2003). Different frequencies of Streptococcus anginosus infection in oral cancer and esophageal cancer. Cancer Science, 94, 492-496. https://doi.org/10.1111/j.1349-7006.2003. tb01471.x

Mougeot, J. L., Stevens, C. B., Cotton, S. L., Morton, D. S., Krishnan, K., Brennan, M. T., ... Bahrani Mougeot, F. K. (2016). Concordance of HOMIM and HOMINGS technologies in the microbiome analysis of clinical samples. Journal of Oral Microbiology, 8, 30379. https://doi. org/10.3402/jom.v8.30379

Mukherjee, P. K., Wang, H., Retuerto, M., Zhang, H., Burkey, B., Ghannoum, M. A., \& Eng, C. (2017). Bacteriome and mycobiome associations in oral tongue cancer. Oncotarget, 8 , 97273-97289.

Nagy, K. N., Sonkodi, I., Szoke, I., Nagy, E., \& Newman, H. N. (1998). The microflora associated with human oral carcinomas. Oral Oncology, 34, 304-308. https://doi.org/10.1016/S1368-8375(98)80012-2

Nash, A. K., Auchtung, T. A., Wong, M. C., Smith, D. P., Gesell, J. R., Ross, M. C., ... Petrosino, J. F. (2017). The gut mycobiome of the Human Microbiome Project healthy cohort. Microbiome, 5, 153. https://doi. org/10.1186/s40168-017-0373-4

Odintsova, V., Tyakht, A., \& Alexeev, D. (2017). Guidelines to statistical analysis of microbial composition data inferred from metagenomic sequencing. Current Issues in Molecular Biology, 24, 17-36. https://doi. org/10.21775/cimb.024.017
Paramsothy, S., Kamm, M. A., Kaakoush, N. O., Walsh, A. J., van den Bogaerde, J., Samuel, D., ... Borody, T. J. (2017). Multidonor intensive faecal microbiota transplantation for active ulcerative colitis: A randomised placebo-controlled trial. Lancet, 389, 1218-1228. https ://doi.org/10.1016/S0140-6736(17)30182-4

Perera, M., Al-Hebshi, N. N., Perera, I., Ipe, D., Ulett, G. C., Speicher, D. J., ... Johnson, N. W. (2017). A dysbiotic mycobiome dominated by Candida albicans is identified within oral squamous-cell carcinomas. Journal of Oral Microbiology, 9, 1385369. https://doi. org/10.1080/20002297.2017.1385369

Perera, M., Al-Hebshi, N. N., Perera, I., Ipe, D., Ulett, G. C., Speicher, D. J., ... Johnson, N. W. (2018). Inflammatory bacteriome and oral squamous cell carcinoma. Journal of Dental Research, 97, 725-732. https:// doi.org/10.1177/0022034518767118

Peters, B. A., Wu, J., Pei, Z., Yang, L., Purdue, M. P., Freedman, N. D., ... Ahn, J. (2017). Oral microbiome composition reflects prospective risk for esophageal cancers. Cancer Research, 77, 6777-6787. https:// doi.org/10.1158/0008-5472.CAN-17-1296

Pushalkar, S., Ji, X., Li, Y., Estilo, C., Yegnanarayana, R., Singh, B., ... Saxena, D. (2012). Comparison of oral microbiota in tumor and nontumor tissues of patients with oral squamous cell carcinoma. BMC Microbiology, 12, 144. https://doi.org/10.1186/1471-2180-12-144

Pushalkar, S., Mane, S. P., Ji, X., Li, Y., Evans, C., Crasta, O. R., ... Saxena, D. (2011). Microbial diversity in saliva of oral squamous cell carcinoma. FEMS Immunology and Medical Microbiology, 61, 269-277. https://doi. org/10.1111/j.1574-695X.2010.00773.x

Sasaki, M., Yamaura, C., Ohara-Nemoto, Y., Tajika, S., Kodama, Y., Ohya, T., ... Kimura, S. (2005). Streptococcus anginosus infection in oral cancer and its infection route. Oral Diseases, 11, 151-156. https:// doi.org/10.1111/j.1601-0825.2005.01051.x

Schmidt, B. L., Kuczynski, J., Bhattacharya, A., Huey, B., Corby, P. M., Queiroz, E. L., ... Muy-Teck, T. (2014). Changes in abundance of oral microbiota associated with oral cancer. PLoS ONE, 9, e98741. https:// doi.org/10.1371/journal.pone.0098741

Seminario-Amez, M., Lopez-Lopez, J., Estrugo-Devesa, A., AyusoMontero, R., \& Jane-Salas, E. (2017). Probiotics and oral health: A systematic review. Medicina Oral Patología Oral y Cirugia Bucal, 22, e282-e288.

Shin, J. M., Luo, T., Kamarajan, P., Fenno, J. C., Rickard, A. H., \& Kapila, Y. L. (2017). Microbial communities associated with primary and metastatic head and neck squamous cell carcinoma - A high fusobacterial and low streptococcal signature. Scientific Reports, 7, 9934. https:// doi.org/10.1038/s41598-017-09786-x

Smittenaar, C. R., Petersen, K. A., Stewart, K., \& Moitt, N. (2016). Cancer incidence and mortality projections in the UK until 2035. British Journal of Cancer, 115, 1147-1155. https://doi.org/10.1038/ bjc. 2016.304

Tateda, M., Shiga, K., Saijo, S., Sone, M., Hori, T., Yokoyama, J., ... Miyagi, T. (2000). Streptococcus anginosus in head and neck squamous cell carcinoma: Implication in carcinogenesis. International Journal of Molecular Medicine, 6, 699-703.

Thompson-Chagoyan, O. C., Maldonado, J., \& Gil, A. (2005). Aetiology of inflammatory bowel disease (IBD): Role of intestinal microbiota and gut-associated lymphoid tissue immune response. Clinical Nutrition, 24, 339-352. https://doi.org/10.1016/j. clnu.2005.02.009

Virgin, H. W. (2014). The virome in mammalian physiology and disease. Cell, 157, 142-150. https://doi.org/10.1016/j.cell.2014.02.032

Wang, H., Funchain, P., Bebek, G., Altemus, J., Zhang, H., Niazi, F., ... Eng, C. (2017). Microbiomic differences in tumor and paired-normal tissue in head and neck squamous cell carcinomas. Genome Medicine, 9, 14. https://doi.org/10.1186/s13073-017-0405-5

Wang, K., Lu, W., Tu, Q., Ge, Y., He, J., Zhou, Y., ... Zhou, X. (2016). Preliminary analysis of salivary microbiome and their potential 
roles in oral lichen planus. Scientific Reports, 6, 22943. https://doi. org/10.1038/srep22943

Wang, K., Miao, T., Lu, W., He, J., Cui, B., Li, J., ... Xiao, L. (2015). Analysis of oral microbial community and Th17-associated cytokines in saliva of patients with oral lichen planus. Microbiology and Immunology, 59, 105-113. https://doi.org/10.1111/1348-0421.12232

Wolf, A., Moissl-Eichinger, C., Perras, A., Koskinen, K., Tomazic, P. V., \& Thurnher, D. (2017). The salivary microbiome as an indicator of carcinogenesis in patients with oropharyngeal squamous cell carcinoma: A pilot study. Scientific Reports, 7, 5867. https://doi.org/10.1038/ s41598-017-06361-2

Yang, S. F., Huang, H. D., Fan, W. L., Jong, Y. J., Chen, M. K., Huang, C. N., ... Su, S. C. (2018). Compositional and functional variations of oral microbiota associated with the mutational changes in oral cancer. Oral Oncology, 77, 1-8. https://doi.org/10.1016/j.oraloncolo gy.2017.12.005

Yang, C. Y., Yeh, Y. M., Yu, H. Y., Chin, C. Y., Hsu, C. W., Liu, H., ... Chang, Y. L. (2018). Oral microbiota community dynamics associated with oral squamous cell carcinoma staging. Frontiers in Microbiology, 9, 862. https://doi.org/10.3389/fmicb.2018.00862

Zhang, M., Sun, K., Wu, Y., Yang, Y., Tso, P., \& Wu, Z. (2017). Interactions between intestinal microbiota and host immune response in inflammatory bowel disease. Frontiers in Immunology, 8, 942. https:// doi.org/10.3389/fimmu.2017.00942

Zhao, H., Chu, M., Huang, Z., Yang, X., Ran, S., Hu, B., ... Liang, J. (2017). Variations in oral microbiota associated with oral cancer. Scientific Reports, 7, 11773. https://doi.org/10.1038/s41598-017-11779-9

\section{SUPPORTING INFORMATION}

Additional supporting information may be found online in the Supporting Information section at the end of the article.

How to cite this article: Robledo-Sierra J, Ben-Amy DP, Varoni E, et al. World Workshop on Oral Medicine VII: Targeting the oral microbiome Part 2: Current knowledge on malignant and potentially malignant oral disorders. Oral Dis. 2019;25(Suppl. 1):28-48. https://doi.org/10.1111/odi.13107 